\title{
Conjugate alkynylation of electrophilic double bonds. From regioselectivity to enantioselectivity
}

\author{
Gonzalo Blay*a \\ José R. Pedro*a \\ Amparo Sanz-Marco a \\ a Departament de Química Orgànica, Facultat de Química, \\ Universitat de València, C/ Dr. Moliner 50, 46100-Burjassot \\ (València), Spain \\ gonzalo.blay@uv.es, josé.r.pedro@uv.es
}

Dedicated to the memory of Professor Aede de Groot

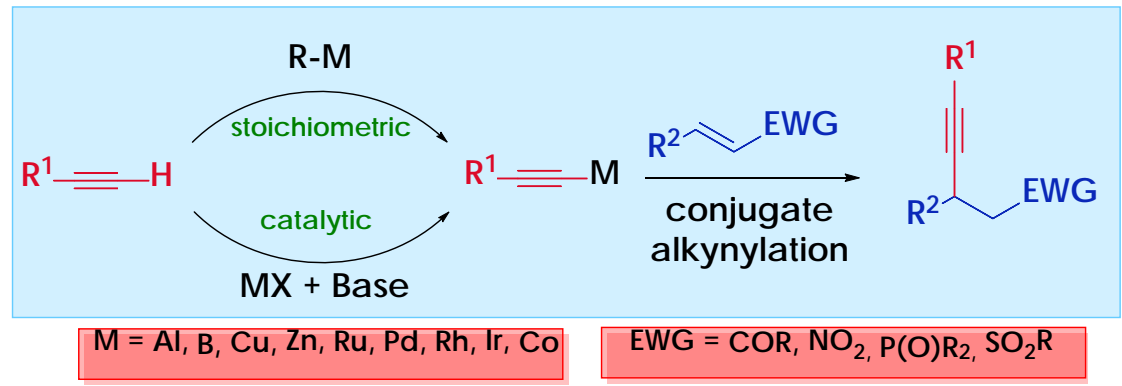

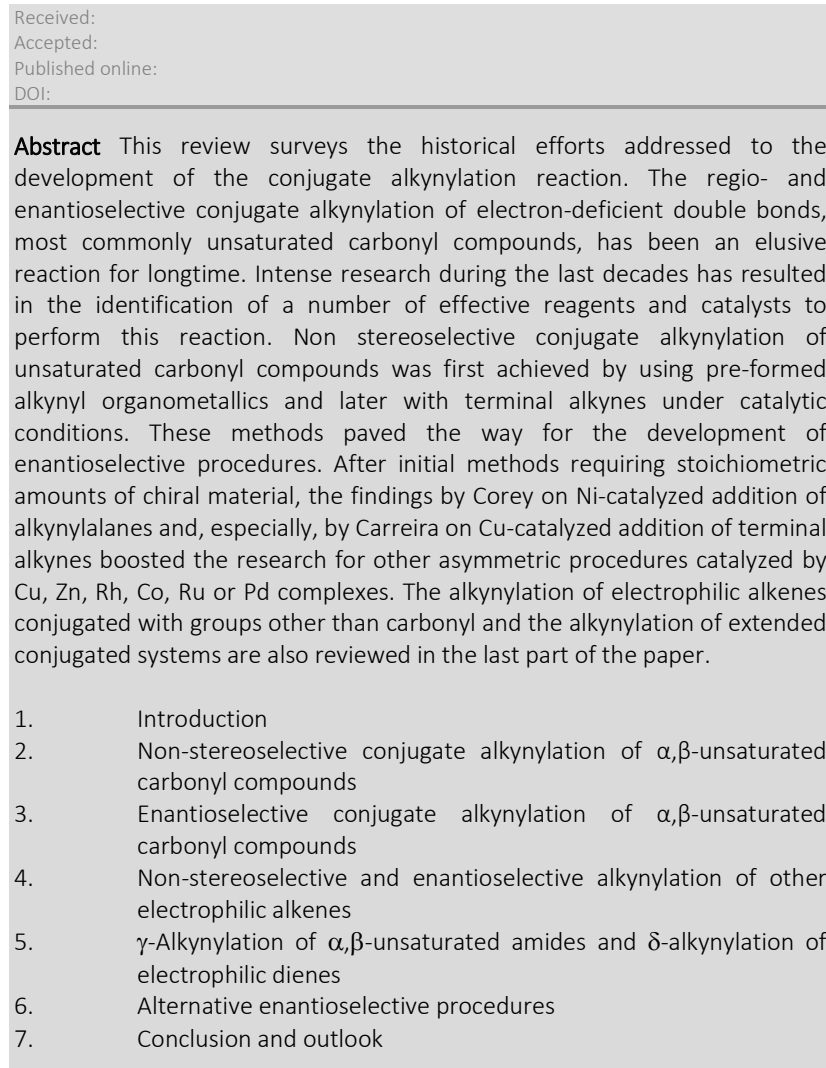

Key words Conjugate addition, alkynes, enones, C-C bond formation, asymmetric catalysis, regioselectivity, electrophilic alkenes

\section{Introduction}

Alkynes are found in nature, 1 more than one thousand compounds featuring a $\mathrm{C}-\mathrm{C}$ triple bond having been isolated from natural sources. The triple bond is also found in many organic molecules of interest in biochemistry and material science. ${ }^{2}$ Furthermore, alkynes are versatile building blocks in synthetic organic chemistry due to the broad spectrum of possibilities for transforming the $\mathrm{C}-\mathrm{C}$ triple bond into other functional groups. The high degree of unsaturation of alkynes increases their reactivity toward electrophilic addition. Accordingly, they can easily undergo hydrogenation, halogenation, hydroboration, hydrosilylation or hydrometalation reactions, among other. Oxidation can lead to hydroxyketones, which can be followed of $\mathrm{C}-\mathrm{C}$ bond cleavage to give acids. Alkynes can also participate in cycloaddition reactions. Many of these reactions are catalyzed through the use of transition metal catalysts. ${ }^{3}$

On the other hand, due to the increased s-character, terminal alkynes are more acidic than other more saturated hydrocarbons such as alkenes and alkanes. Thus, they can be deprotonated under different basic conditions to give nucleophilic metal alkynylides, which can react with carbonbased electrophiles to give internal alkynes with concomitant formation of a new $\mathrm{C}-\mathrm{C}$ bond. Deprotonation of terminal alkynes can be achieved upon treatment with a strong base such as lithium amide, butyllithium, or Grignard or zinc reagents, generating metal alkynylides stoichiometrically, which can be transmetalated to other metal alkynylides if convenient (Scheme 1, path a). On the other hand, metal alkynylides can be produced with much weaker bases in the presence of a catalytic amount of a transition metal with high affinity towards $\pi$ bonding to $\mathrm{C}-\mathrm{C}$ triple bonds. Coordination of the metal to the triple bond can increase the acidity of the terminal proton in such a way that it can be deprotonated by mild bases such as tertiary amines (Scheme 1, path b). In general, the alkynylides formed with this last procedure are less reactive than those prepared in a stoichiometric fashion with respect to addition reactions. 


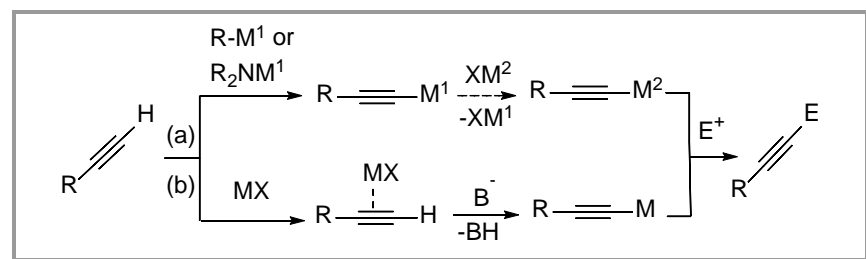

Scheme 1 Generation of metal alkynylides from terminal alkynes. (a) Stoichiometric procedure. (b) Catalytic procedure

In recent years, the nucleophilic addition of metal alkynylides generated by any of these methods to prochiral electrophiles has emerged as one of the most efficient methodologies for the synthesis of internal alkynes bearing a propargylic stereogenic center. Thus, considerable success has been obtained in the enantioselective alkynylation of carbonyl compounds 4 and imines $^{5}$ to give propargylic alcohols and amines, respectively. However, the regioselective conjugate alkynylation of electrophilic double bonds conjugated with electronwithdrawing groups, especially $\alpha, \beta$-unsaturated carbonyl compounds, has supposed a formidable challenge, even in a non-enantioselective manner. ${ }^{6}$ This has been due in part to the fact that alkynyllithium and alkynyl Grignard reagents give preferential 1,2-atack with conjugated carbonyl compounds. ${ }^{7}$ Furthermore, despite alkyl or aryl copper (I) reagents have been widely used to achieve the regioselective conjugate alkylation or arylation of enones, the use of related alkynylcuprates in conjugate additions has been hampered by the inability of these reagents to transfer the alkyne. In fact, the high tenacity with which copper binds alkynyl groups has been exploited in the design of mixed cuprate reagents where an alkynyl ligand serves as non-transferrable dummy ligand to ensure selective group transfer.

Over the last years, many efforts have been devoted to overcome these drawbacks, which have led to the identification of different effective reagents and catalysts to achieve the conjugate alkynylation of electrophilic alkenes. In this review we will survey the most relevant literature related with this elusive reaction, with especial emphasis in the development of enantioselective procedures with $\beta$-substituted $\alpha, \beta$-unsaturated carbonyl compounds (Scheme 2).

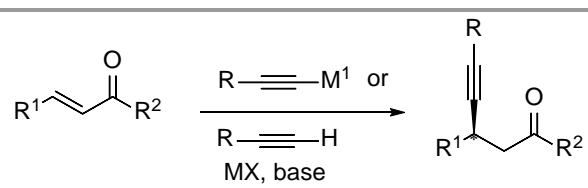

Scheme 2 Conjugate alkynylation of $\alpha, \beta$-unsaturated carbonyl compounds

\section{Non-stereoselective conjugate alkynylation of $\alpha, \beta$-unsaturated carbonyl compounds}

\subsection{Conjugate alkynylation with metal alkynylides}

\subsubsection{Conjugate alkynylation with aluminum alkynylides}

The first conjugate alkynylation of enones was reported in 1971 by Hooz, using alkynylalanes prepared by converting a terminal alkyne into the lithium derivative (using $n$-butyllithium) followed by transmetalation after addition of diethylaluminum chloride (Scheme 3). ${ }^{8}$ The reaction showed a strong solvent effect and the best results were usually obtained in etherligroin, although each reaction required a proper choice of variables. Only enones that could afford the $s$-cis conformation underwent the conjugate alkynylation, while s-trans conformationally restricted enones; i. e. cyclic enones, preferred the 1,2-alkynylation pathway. To explain these results, the authors proposed the intramolecular delivery of the alkynyl group through a six-membered transition state with the $\mathrm{Al}$ atom coordinated to the carbonyl group, which would be very constrained for transoid enones. Enals and alkynones also gave the 1,2-addition products.

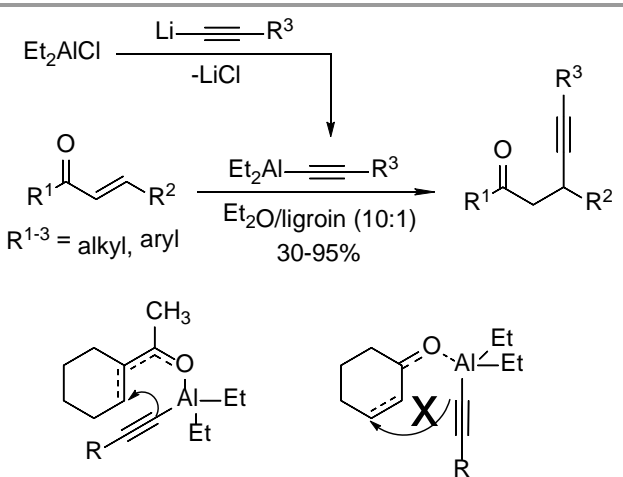

Scheme 3 First conjugate alkynylation of enones with diethylalkynylalanes. Proposed transition states for s-cis and s-trans enones.

Soon later, Pappo and Collins reported that tri-1octynylaluminum (and also tri-1-octynylboron), in spite of their high instability, gave conjugate addition to a 4-hydroxy-2cyclopentenone derivative. ${ }^{9}$ The fact that the alkyne group added cis to the $\mathrm{OH}$ seemed to indicate the participation of this group, probably through a cyclic intermediate. Furthermore, protection of the $\mathrm{OH}$ prevented the 1,4-addition. The reaction also worked with the analogous trialkynylgallium reagent, although in this case the ratio of diastereomers was inverted. The same authors also reported the addition of a trialkynylboron derivative to methyl vinyl ketone in a synthesis of 16-hydroxy analogs of PGE2. ${ }^{\text {b }}$ Unfortunately, these reactions utilized only one of the three acetylene groups available, what constituted a serious disadvantage (Scheme 4).

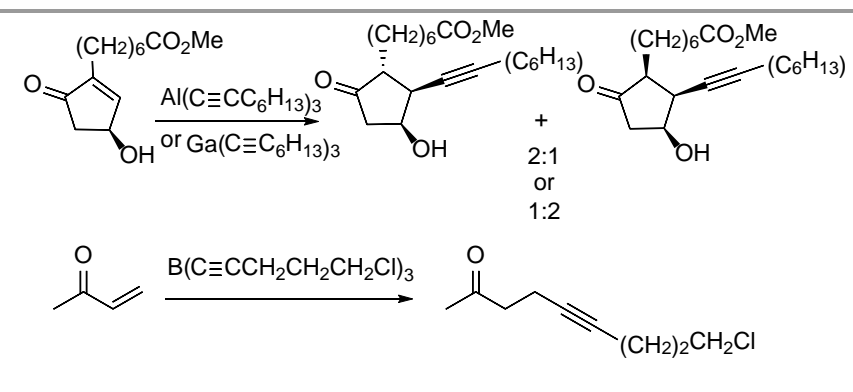

Scheme 4 Conjugate alkynylation with trialkynylaluminum, trialkynylgallium or trialkynylboron reagents by Pappo and Collins

The first example of conjugate alkynylation of cyclic enones was reported by Schwartz in 1978. The reaction was carried out by using alkynylalanes in the presence of a $\mathrm{Ni}(\mathrm{I})$ catalyst prepared after reduction of $\mathrm{Ni}(\mathrm{acac})_{2}$ (acac = acetylacetonate) with diisobutylaluminum hydride (DIBAL-H) (Scheme 5).10 The 
conditions were applied to a number of 2-cyclopentenone and 2-cyclohexenone derivatives. In the case of $\gamma$-substituted enones, the trans isomer was obtained exclusively. The reaction was also performed with methyl vinyl ketone. The use of excess of alkynylalane was mandatory to minimize the aldol condensation of the resulting aluminum enolate with unreacted enone. ${ }^{10 \mathrm{~b}}$

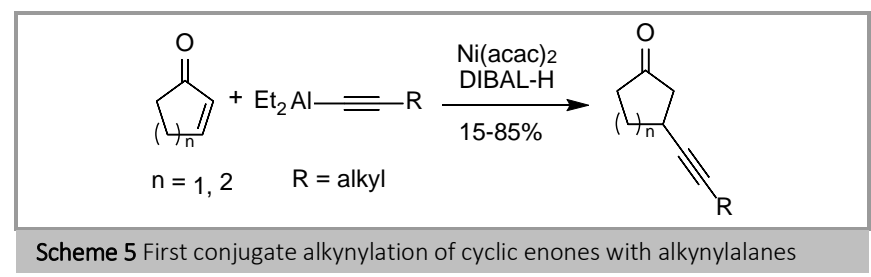

Yamamoto also described the conjugate alkynylation of 2cyclopentenone, 2-cyclohexenone and chalcone involving organoaluminum species generated from alkynyllithium reagents and aluminum tris(2,6-diphenylphenoxide). ${ }^{11}$

In 2014, Fillion and Ahmar described the first conjugate alkynylation of $\beta, \beta$-disubstituted conjugated carbonyl compounds, generating an all carbon substituted quaternary carbon. The reaction involved the addition of alkynylalanes or alkynyl Grignard reagents to doubly activated alkenes derived from Meldrum's acid. Bulky groups such as 2-naphthyl or orthosubstituted aryl rings attached to the double bond prevented the reaction indicating the importance of steric effects. Silverpromoted cyclization of the resulting $\beta$-alkynyl enones provided $\gamma$-alkylidene butyrolactones bearing a quaternary carbon (Scheme 6). ${ }^{12}$

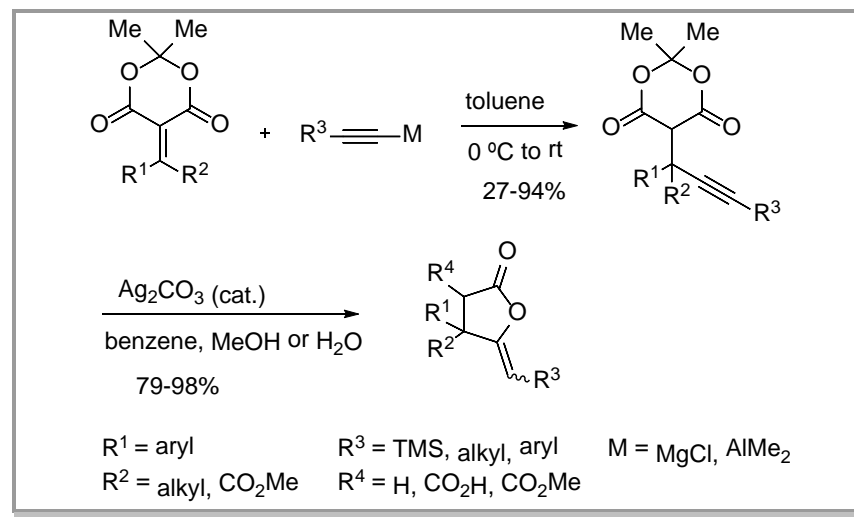

Scheme 6 First conjugate alkynylation of $\beta, \beta$-disubstituted conjugated carbonyl compounds

\subsubsection{Conjugate alkynylation with boron alkynylides}

In 1977, Brown employed B-1-alkynyl-9-borabicyclo[3.3.1] nonanes, readily prepared by the reaction of boron trifluoride diethyl etherate with the corresponding lithium methyl alkynyldialkylborinate, to achieve the conjugate alkynylation of methyl vinyl ketone and related enones, with high yields. Although the reaction was quite general, it was restricted to enones that were able to attain the s-cis conformation (Scheme 7). 13

In 2009, based on work by Chong with (1alkynyl)diisopropoxyboranes (see section 3.1), Woodward introduced potassium alkynyltrifluoroborates as pronucleophiles for the conjugate alkynylation of acyclic enones.
The reaction involved the abstraction of a fluoride from the potassium trifluoroborate by means of $\mathrm{BF}_{3} \cdot \mathrm{Et}_{2} \mathrm{O}$ leading to alkynyldifluroboranes which reacted quickly with the electrophile due to the high acidity of the boron atom and its capability to coordinate with the carbonyl group (Scheme 8).14

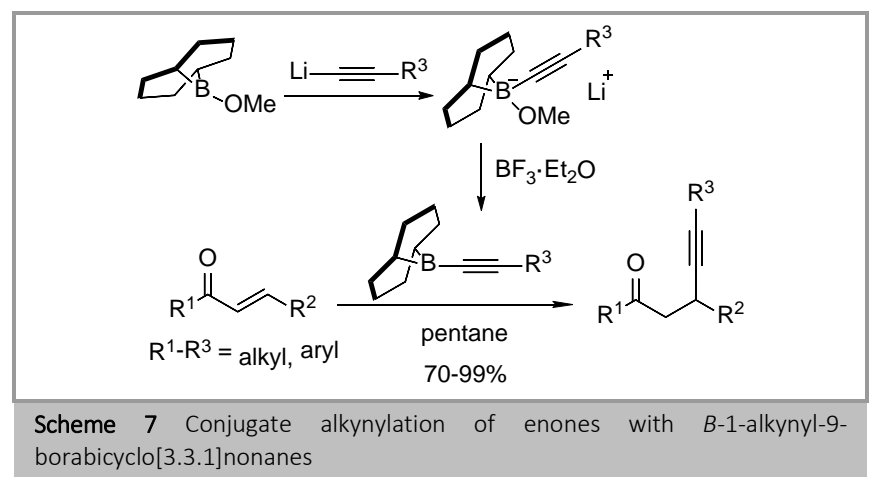

$$
\begin{array}{ll} 
& \\
\mathrm{R}^{1}=\mathrm{Me}, \mathrm{Et}, \mathrm{Ph} & \mathrm{RF}_{3} \mathrm{R}_{3} \cdot \mathrm{Et}_{2} \mathrm{O}, \mathrm{CH}_{2} \mathrm{Cl}_{2} \\
\mathrm{R}^{2} & =\text { alkyl, } \mathrm{Ph} \\
\mathrm{R}^{3} & =\text { alkyl, } \mathrm{Ph}, \text { propylydene }
\end{array}
$$

Scheme 8 Alkynyl trifluoroborates as nucleophiles for the conjugate alkynylation of enones

\subsubsection{Conjugate alkynylation with zinc or copper alkynylides}

Electrophilic silicon reagents have been used as promoters of conjugate alkynylation by zinc and copper reagents. Thus, Kim reported in 1990 the conjugate alkynylation of enones using alkynylzinc compounds in the presence of $t$-butyldimethylsilyl triflate (TBSOTf) at $-40{ }^{\circ} \mathrm{C} .15$ The method could be applied to both s-cis and s-trans enones, with consistent yields for $\beta$ monosubstituted enones. However, $\beta$-unsubstituted enones gave lower yield due to conjugate addition of the resulting enolate with unreacted enone, while $\beta, \beta$-disubstituted enones were poorly reactive and gave low yields of 1,2- and 1,4addition products (Scheme 9). The same authors demonstrated that tert-butyldimethylsilyl triflate also promoted the addition of lithium alkynylcuprate reagents, $\mathrm{RC} \equiv \mathrm{CCuLiCN}$, (derived from 1-hexyne, trimethylsilylacetylene or phenylacetylene) to $\beta$ monosubstituted cyclic and acyclic $\alpha, \beta$-enones. The use of etherdioxane as solvent minimized the 1,2 -addition. ${ }^{16}$

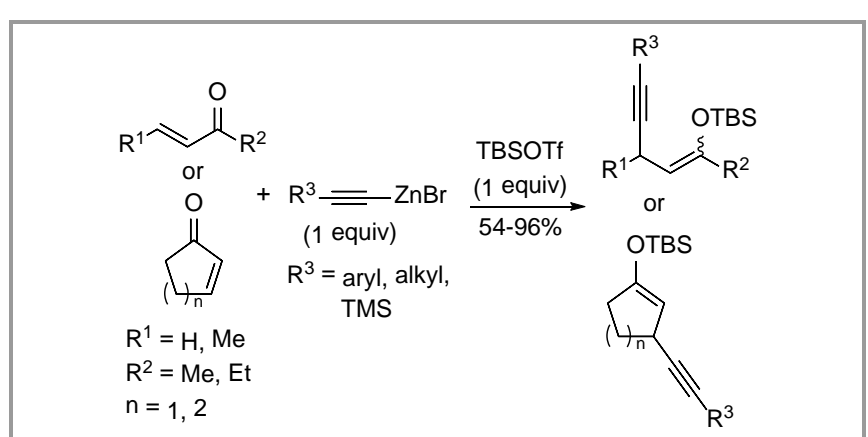

Scheme 9 Conjugate alkynylation of enones with alkynylzinc reagents promoted by $t$-butyldimethylsilyl triflate 
Later, Nilsson reported that copper acetylide reagents added to enones present as s-trans conformers to provide good yields of the silyl enol ethers in the presence of TMSI (TMS = trimethylsilyl) and lithium iodide in tetrahydrofuran. Typically good substrates were 2-cyclopentenone, 2-cyclohexenone, $\alpha, \beta$ unsaturated aldehydes, and $\beta$-alkoxy- $\alpha$-enones. Copper reagents prepared from $\mathrm{CuI}$ and an alkynyllithium gave considerably higher yields than those prepared from $\mathrm{CuBr}$ or CuCN (Scheme 10). ${ }^{17}$

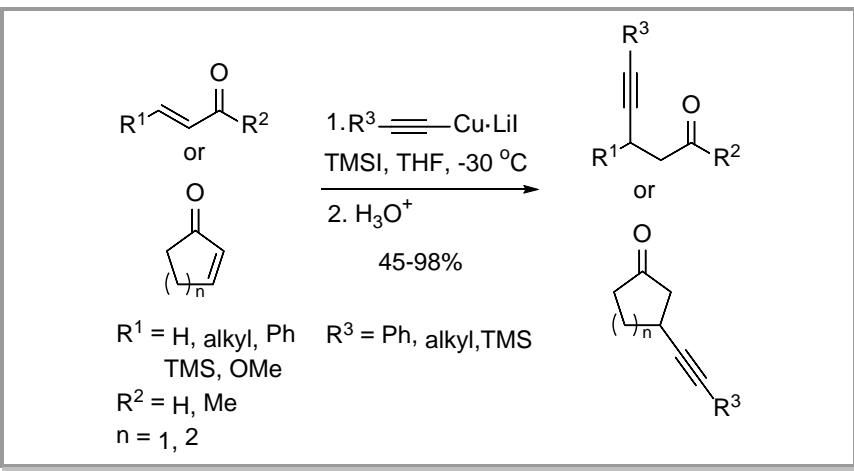

Scheme 10 Conjugate alkynylation of enones with copper acetylides promoted by iodotrimethylsilane and lithium iodide in tetrahydrofuran

This procedure has been employed by Aggarwal in a recent synthesis of the veterinary drug alphaprostol and prostaglandine $\mathrm{PGF}_{2 \alpha}$ (Scheme 11). ${ }^{18}$

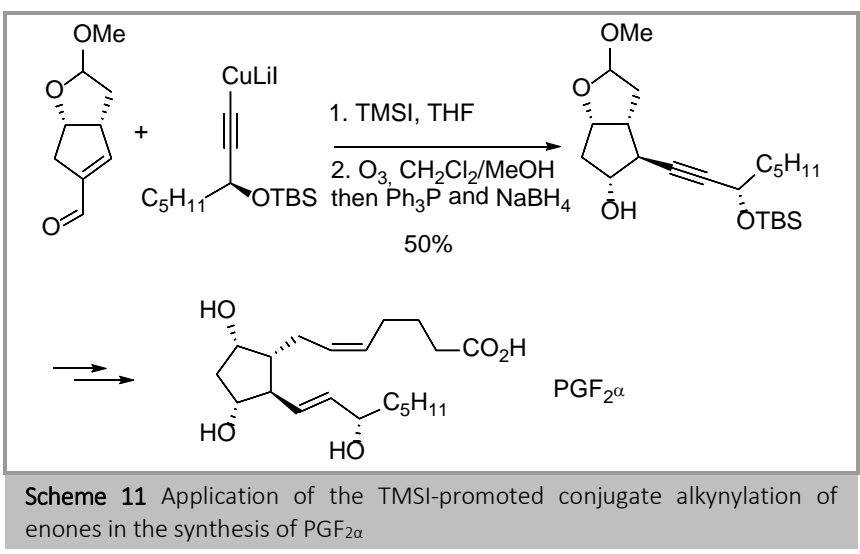

Despite the claimed low nucleophilicity of alkynylcopper species, Gabbutt was able to achieve the conjugate alkynylation of 3-formyl chromones with lithium dialkynylcuprates, $(\mathrm{RC} \equiv \mathrm{C})_{2} \mathrm{CuLi}$, to give alkynylated chromanones. These compounds exhibited a marked instability to acid and isomerized under prolonged contact with silica gel to conjugated enynones (Scheme 12). ${ }^{19}$

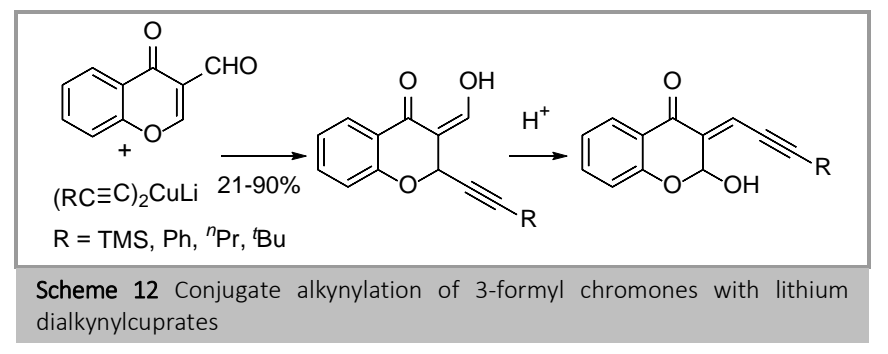

\subsubsection{Alkynylsilanes and other}

Alkynylsilanes have been used by Su and col. as nucleophiles for the conjugate alkynylation of acrylates. The reaction was catalyzed by $\mathrm{InCl}_{3}$ and afforded the corresponding products with yields above 75\% (Scheme 13). ${ }^{20}$ Different alkynes were examined. Among (trimethylsilyl)phenylacetylene derivatives, trimethyl[(4-methoxyphenyl)ethynyl]silane gave the best result. However, alkynylsilanes bearing a strong electron-withdrawing group on the benzene ring failed to react. Alkyl derivatives such as trimethyl(1-hexynyl)silane reacted with ethyl acrylate with good yield but other alkynes such as 1-cyclohexenylethyne or 3,3-dimethyl-1-butyne did not react.

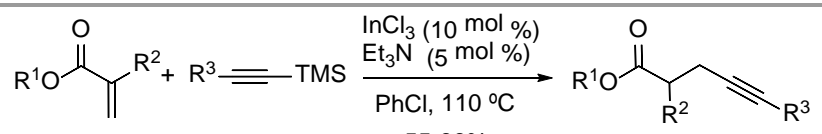

$$
\begin{aligned}
& \mathrm{R}^{1}=\text { alkyl } \quad \mathrm{R}^{3}=\text { alkyl, aryl } \quad 55-96 \% \\
& \mathrm{R}^{2}=\mathrm{H}, \mathrm{Me}
\end{aligned}
$$

Scheme 13 Conjugate alkynylation of acrylates with trimethylsilylalkynes

Finally, other metal alkynylides have been tested for conjugate alkynylation during the course of organometallic research. For instance, Shibata and Baba developed organotantalum reagents from organotin compounds and $\mathrm{TaCl}_{5}$ which reacted with enones to give the conjugate addition products. The authors reported just three examples involving alkynylation. ${ }^{21}$

\subsection{Conjugate alkynylation with terminal alkynes}

\subsubsection{Rhodium-catalyzed conjugate alkynylation}

In 1990 Kovalev et al. reported the first conjugate alkynylation using terminal alkynes as nucleophiles. The $\left[\mathrm{RhCl}\left(\mathrm{PMe}_{3}\right)_{3}\right]$ complex in acetone catalyzed the addition of 2-alkyl- and 2-arylacetylenes to vinyl ketones with good yields at room temperature (Scheme 14). ${ }^{22}$ Higher temperatures reduced the yield of the reaction product due to a by-process of dehydrodimerization of the alkynes. However, the reaction had some drawbacks such as long reaction times and the use of a non-commercial catalyst that required trimethylphosphine, a volatile and toxic chemical, for its synthesis. To avoid these drawbacks, Lerum and Chisholm performed the reaction in the presence of $\left[\mathrm{Rh}(\mathrm{acac})(\mathrm{CO})_{2}\right]$, a stable rhodium complex, and tris(o-methoxyphenyl)phosphine at reflux in benzene. ${ }^{23}$ Phenylacetylene as well as functionalized alkylacetylenes reacted with $\beta$-unsubstituted enones in fair to good yields, with lower reaction times than those reported by Kovalev.

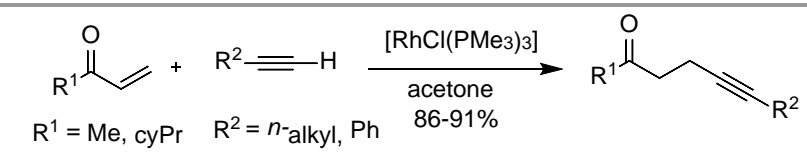

Scheme 14 Rh-catalyzed conjugate alkynylation of vinyl ketones with terminal alkynes

\subsubsection{Ruthenium-catalyzed conjugate alkynylation}

Conjugate alkynylation of vinyl ketones with terminal alkynes has been also achieved with $\mathrm{Ru}$ catalysis. Dixneuf reported the use of the $\left[\mathrm{Ru}\left(\mathrm{O}_{2} \mathrm{CH}\right)(\mathrm{CO})_{2}\left(\mathrm{PPh}_{3}\right)\right]_{2}$ complex for the alkynylation 
of butenone obtaining low yields below $50 \%$ with most of the aliphatic and aromatic alkynes tested, except with phenylacetylene. ${ }^{24}$ Chang reported the 1,4-addition of a wide range of terminal alkynes, mostly alkyl-substituted, to vinyl ketones by combining $\left[\mathrm{RuCl}_{2}(p \text {-cymene })\right]_{2}$ and pyrrolidine, with good yields in most of the cases (Scheme 15). ${ }^{25} \mathrm{~A}$ diverse range of functional groups were compatible with the reaction conditions.

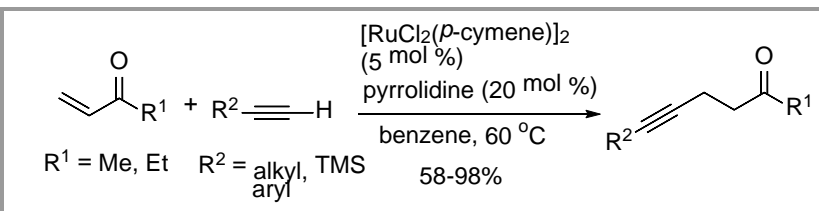

Scheme 15 Ru-catalyzed conjugate alkynylation of vinyl ketones with terminal alkynes

On the other hand, the group of Uemura developed a procedure for the conjugate alkynylation of ethyl acrylate with terminal alkynes in the presence of $\mathrm{Ru}_{3}(\mathrm{CO})_{12}$ and bis(triphenylphosphine)iminium chloride ([PPN]Cl), as a chloride ion source, in $\mathrm{N}$-methylpyrrolidinone (NMP) at $60{ }^{\circ} \mathrm{C}$. Aromatic alkynes reacted with fair to good yields, higher with electron-donating substituents attached to the phenyl group. Trimethylsilylacetylene and 1-octyne reacted slower and gave the alkynylation products with low yields. ${ }^{26 a}$ Later, these authors extended the reaction to enones and to ethyl 2butynoate. Furthermore, the authors found that in the presence of LiI instead of [PPN]Cl the reaction led to conjugate dienes instead of the expected $\beta$-alkynyl esters (Scheme 16). ${ }^{26 \mathrm{~b}}$

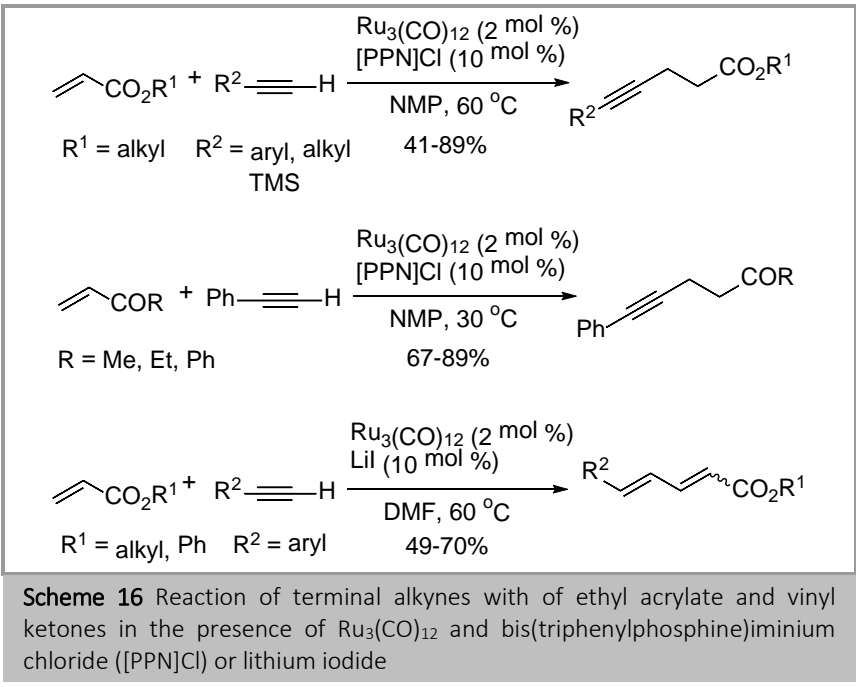

In 2012, Ito et al. reported a procedure for the conjugate addition of terminal alkynes (arylacetylenes and trimethylsilylacetylene) to a variety of $\alpha, \beta$-unsaturated carbonyl compounds (ketones, esters, amides and a phosphonate) under catalysis with a $\mathrm{Ru}(\mathrm{II})$-phebox complex (phebox = phenyl bisoxazoline). The addition of arylalkynes and trimethylsilylacetylene to methyl vinyl ketone, phenyl vinyl ketone, alkyl acrylates and acrylamides took place with good yields. However cyclohexylacetylene gave low yields with ketones and with acrylic esters (Scheme 17). ${ }^{27}$

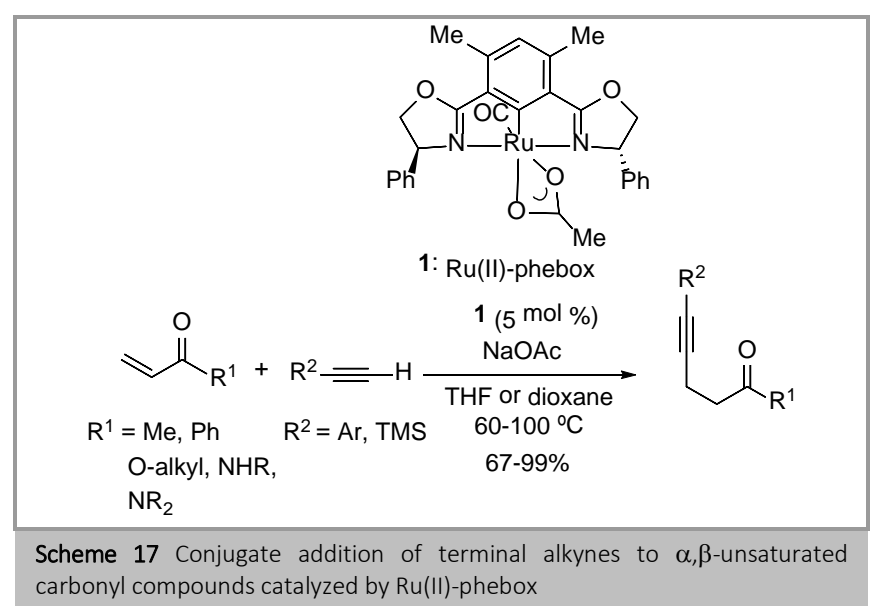

\subsubsection{Palladium-catalyzed conjugate alkynylation}

Chen and Li described in 2004 the first Pd-catalyzed conjugate alkynylation of vinyl ketones using $\mathrm{Pd}(\mathrm{OAc})_{2} / \mathrm{PMe}_{3}$ in water or acetone, obtaining fair to good yields in the addition of several aryl and alkyl acetylenes to methyl or ethyl vinyl ketone. ${ }^{28} \mathrm{~A}$ modification of the catalyst substituting $\mathrm{PMe}_{3}$ by 1,3-dimesityl$1 \mathrm{H}$-imidazol-3-ium chloride, a NHC ligand, permitted to carry out the addition of arylacetylenes to acrylates (Scheme 18).

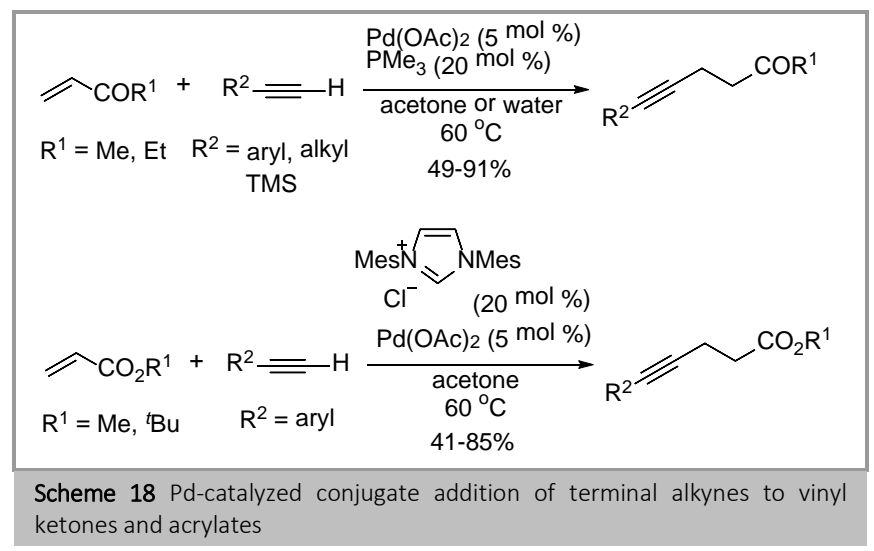

In 2012, the group of Mascareñas described a new Pd-catalyzed conjugate alkynylation that could be applied to $\beta$-substituted conjugate carbonyl compounds. A Pd(0) complex formed from $\left[\mathrm{Pd}_{2}(\mathrm{dba})_{3}\right]$ and (2,4-di-tert-butylphenyl) phosphite catalyzed the reaction of triisopropylsilylacetylene with cyclic and linear enones to give the alkynylation products with good yields (3894\%) (Scheme 19). ${ }^{29}$

Scheme 19 Pd-catalyzed conjugate alkynylation of $\beta$-substituted enones


However, a $\beta, \beta$-disubstituted enone did not react under these conditions. The reaction was also possible with aryl-substituted alkynes or with alkynes substituted with bulky aliphatic groups, although in these cases slow addition of the alkyne was required to avoid homodimerization.

\subsubsection{Copper-catalyzed conjugate alkynylation}

In 2003, Carreira reported the first conjugate addition of alkynes catalytic in copper. Alkenes derived from Meldrum's acid were reacted with copper alkynylides generated in situ from terminal alkynes, $\mathrm{Cu}(\mathrm{OAc})_{2}$ and sodium ascorbate as a reductant in a biphasic $\mathrm{H}_{2} \mathrm{O}: \mathrm{t} \mathrm{BuOH}$ medium. In this case, the low nucleophilicity inherent to copper (I) alkynylides was compensated by the double activation of the alkene (Scheme 20). ${ }^{30}$ A variety of substituents, aromatic and heteroaromatic, branched and unbranched aliphatic were tolerated on the acceptor. Importantly, in the case of $\alpha, \beta, \gamma, \delta$-diene acceptors, only 1,4-addition was observed. On the other hand, both electronrich and -poor aromatic groups as well as heteroaromatics were tolerated on the alkyne. Control experiments showed that the ascorbate may play an important role beyond reducing $\mathrm{Cu}(\mathrm{II})$, and that water was essential for the success of the reaction. Interstingly, the reaction products could be readily converted into $\beta$-alkynyl carboxylic acids by simply heating in a DMF/water solution. The catalytic system was also applicable with ethyl propiolate, which is characterized by the low nucleophilicity of metal propiolates and by its excellent properties as Michael acceptor that make it prone to undergo self-addition reactions. ${ }^{30 \mathrm{~b}}$

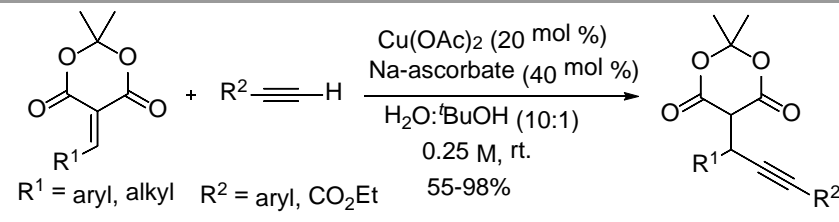

$$
\begin{aligned}
& \text { DMF/ } / \mathrm{H}_{2} \mathrm{O} \\
& \text { (10:1) } \\
& 100{ }^{\circ} \mathrm{C} \\
& \text { 90-91\% } \\
& \overbrace{R^{2}}^{\mathrm{R}^{1}} \\
& \mathrm{R}^{1}={ }^{i} \mathrm{Pr}, \mathrm{R}^{2}=\mathrm{Ph} \\
& \mathrm{R}^{1}=4-\mathrm{MeOC}_{6} \mathrm{H}_{4}, \mathrm{R}^{2}=\mathrm{Ph}
\end{aligned}
$$

Scheme 20 Conjugate alkynylation of Meldrum's acid derived alkenes with terminal alkynes catalyzed by $\mathrm{Cu}(\mathrm{I})$

Based on Carreira's conjugate alkynylation of Meldrum's acid derivatives, Jiao developed a copper/iron co-catalyzed tandem reaction conjugate addition-cyclization-hydrolysisdecarboxylation yielding $\gamma$-alkylidenebutyrolactones, similar to those described in Scheme 6.31

Furthermore, Prajapati has described a three-component tandem conjugative alkynylation/6-endo cyclization sequence to access pyrano[2,3-d]-pyrimidines from barbituric acids, aldehydes and terminal alkynes. The reaction is carried out in the presence of catalytic amounts of $\mathrm{Cu}(\mathrm{OTf})_{2}$ and $t$-BuOK and seems to involve the aldol condensation of the barbiturate and aldehyde catalyzed by the base, followed of conjugate addition of a copper acetylide to the resulting Michael acceptor and copper-catalyzed cyclization. Arylacetylenes bearing electronwithdrawing groups on the aromatic ring gave low yields of cyclization product (Scheme 21).32

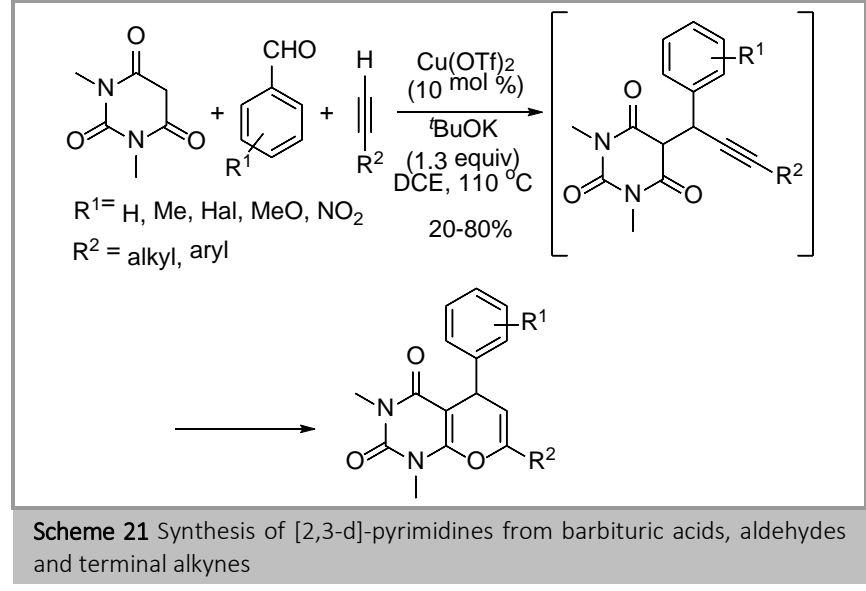

\subsubsection{Zinc-catalyzed conjugate alkynylation}

Finally, Kidway et al. developed a conjugate alkynylation of enones with phenylacetylene or 1-octyne using a [ $\mathrm{Zn}(\mathrm{L}-$ proline)2] complex as catalyst, and triethylamine in water. Other zinc complexes with aminoacids having primary amine moieties were inactive. The $\beta$-alkynyl ketones were obtained with high yields, although in racemic form despite using a chiral catalyst (Scheme 22). ${ }^{33}$

$\mathrm{R}^{3}=\mathrm{Ph}, \mathrm{C}_{6} \mathrm{H}_{13}$
$\mathrm{R}^{2}=\mathrm{H}$, alkyl, aryl
Scheme 22 Conjugate alkynylation of enones by $\mathrm{Zn}$-proline in aqueous
medium

\section{Enantioselective conjugate alkynylation of $\alpha, \beta$-unsaturated carbonyl compounds}

The conjugate alkynylation of $\beta$-substituted prochiral enones gives rise to the generation of a new stereogenic center in propargylic position. Giving the importance of chirality in chemistry, tremendous efforts have been done to achieve this kind of transformations in an enantioselective manner. In this section we will review the literature regarding enantioselective alkynylation. The methods will be classified according the amount of chiral material required as stoichiometric or catalytic procedures.

\subsection{Enantioselective stoichiometric procedures}

The group of Carreño described in 1996 the desymmetrization of $(R)$-[( $p$-tolylsulfonyl)methyl] quinols via a diastereoselective uncatalyzed addition of organoaluminum reagents. The study included some examples with alkynyldimethylaluminum reagents. The authors found that the reaction occurred from the face containing the $\mathrm{OH}$ with total $\pi$-facial diastereoselectivity 
dictated by the sulfonyl moiety, allowing the simultaneous generation of two stereogenic centers (Scheme 23). ${ }^{34}$

$$
\begin{aligned}
& \text { 瓜 } \\
& \mathrm{R}^{1}=\mathrm{H}, \mathrm{Me} \quad \mathrm{R}^{2}=\mathrm{TMS},{ }^{n} \mathrm{Bu}
\end{aligned}
$$

Scheme 23 Desymmetrization of $(R)-[(p$-tolylsulfonyl)methyl]quinols via a diastereoselective uncatalyzed addition of alkynylorganoaluminum reagents

In 2000, Chong developed the first enantioselective conjugate alkynylation of prochiral enones. The authors prepared chiral alkynyl boranes derived from 3,3'-disubstituted 2,2'binaphthols, which reacted enantioselectively with enones to give the corresponding products with good yields and high enantiomeric excesses for a number of substrates and arylacetylenes. Low enantiomeric excesses, below 50\%, were obtained however for enones bearing an alkyl group at the $\beta$ position or with alkylacetylenes. Furthermore, the reaction was restricted to enones that could adopt an s-cis conformation. Similarly, no reaction was observed with a $\beta, \beta$-disubstituted enone. Reactions of $Z$ enones gave essentially the same selectivities than their $E$ counterparts, probably through isomerization of the double bond. The stereochemistry of the reaction was explained on the basis of cyclic six-membered transition state, with the transition state leading to the minor enantiomer would be disfavored due to steric interactions (Scheme 24). ${ }^{35}$

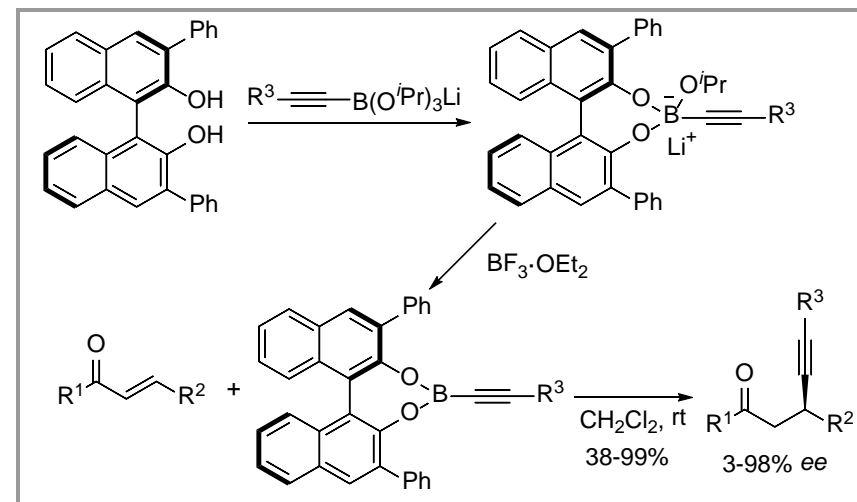

$\mathrm{R}^{1}, \mathrm{R}^{2}=$ alkyl, aryl $\mathrm{R}^{3}=n-\mathrm{C}_{6} \mathrm{H}_{13,}, \mathrm{Ph}$ $\mathrm{BnOCH}_{2}$
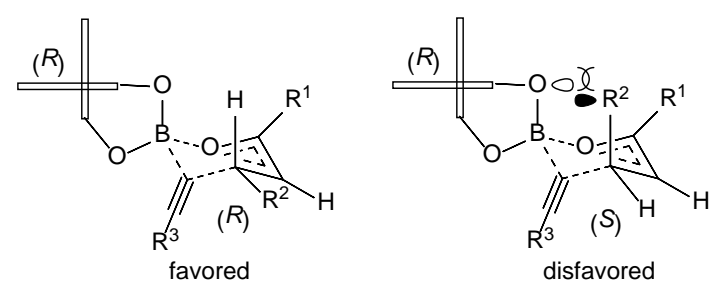

Scheme 24 First enantioselective conjugate alkynylation of enones with chiral akynylborates

A different approach by Carreira involved the use of chiral alkenyl oxazepanediones, prepared from ephedrine, as electrophiles. These chiral doubly activated alkenes reacted with zinc alkynylides generated in situ under mild conditions by the action of $\mathrm{Zn}(\mathrm{OTf})_{2}$ and $\mathrm{Et}_{3} \mathrm{~N}$ on terminal acetylenes to give the corresponding alkynylated products that, after hydrolysis of the chiral auxiliary and decarboxylation, yielded enantiomerically enriched $\beta$-propargylic acids (Scheme 25). The addition was highly stereoselective for acceptors with branched substituents and less for acceptors bearing unbranched alkyl chains. Additions to acceptors with aromatic or unsaturated residues did not proceed. The reaction required a high catalyst load (60 mol \%), which could be lowered to $20 \mathrm{~mol} \%$, when the reaction was conducted at $60{ }^{\circ} \mathrm{C}$ in toluene. This reaction constituted the first example of conjugate addition of terminal alkynes mediated by zinc. ${ }^{36}$

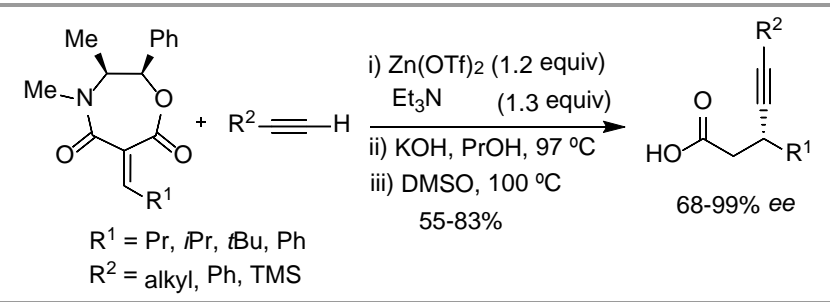

Scheme 25 Enantioselective conjugate alkynylation using ephedrine as chiral auxiliary

Cui and Walker reported in 2010 the conjugate alkynylation of arylidene Meldrum's acid derivatives with excess of $\mathrm{Et}_{2} \mathrm{Zn}$ and $\mathrm{RC} \equiv \mathrm{CMgCl}$ in the presence of trifluoroethanol. The reaction probably involves the participation of alkynylzinc species formed upon transmetalation of the Grignard reagent with a zinc alkoxide generated after fast neutralization of diethylzinc by trifluoroethanol. The reaction was carried out in the presence of 2.9 equivalents of cinchonidine as chiral promoter and the resulting alkynes were obtained usually with high yields and enantiomeric excesses (Scheme 26). ${ }^{37} \mathrm{~A}$ wide range of functional groups were well-tolerated. Acceptors having orthosubstituted aryl groups or aliphatic groups attached to the double bond generally provided moderate enatioselectivities, although, this limitation could be overcome by using ( $r a c$ )Mosher acid instead of trifluoroethanol as the additive. A wide variety of Zn alkynylides possessing aliphatic, aromatic, and silyl groups, could be employed in the reaction with good results.

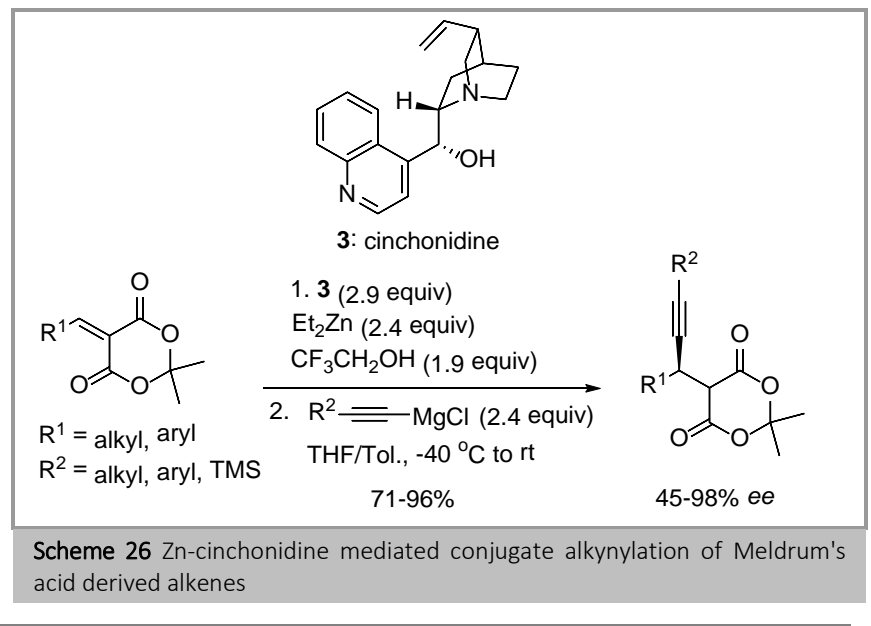


The synthetic usefulness of this reaction was demonstrated with the enantioselective preparation of a GPR40 receptor agonist drug, which is a potential therapeutic target for insulin-involved disorders such as type 2 diabetes (Scheme 27). ${ }^{37 \mathrm{~b}}$

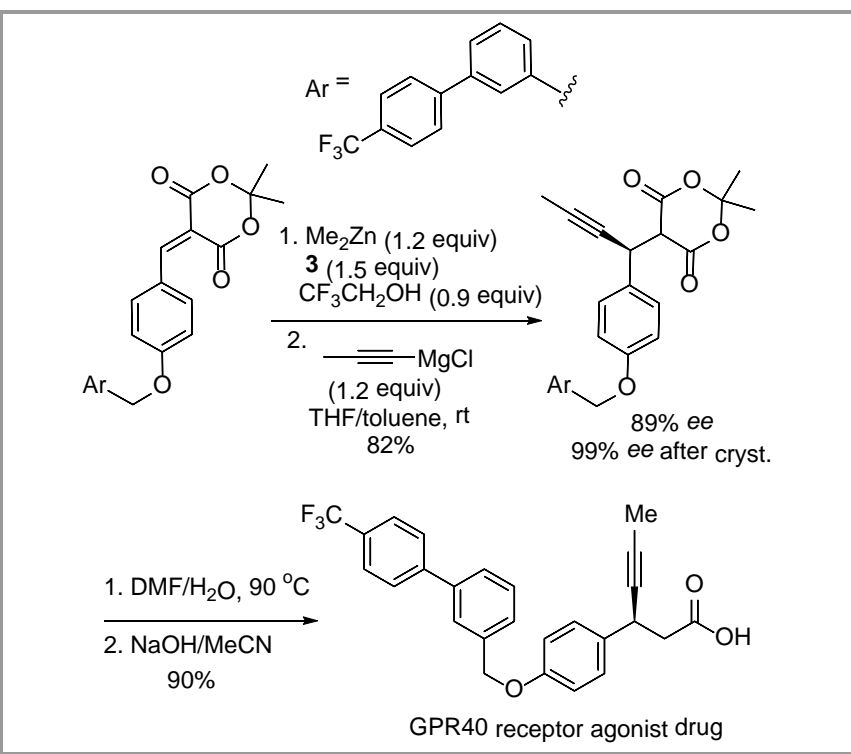

Scheme 27 Application of the conjugate alkynylation of arylidene Meldrum's acid derivatives with $\mathrm{Et}_{2} \mathrm{Zn}$ and $\mathrm{MeC} \equiv \mathrm{CMgCl}$ to the synthesis of GPR40

\subsection{Enantioselective catalytic procedures}

\subsubsection{Nickel catalysis}

In 2004, Corey et al. described the first catalytic enantioselective conjugate alkynylation of enones. ${ }^{38}$ These authors performed the reaction of 2-cyclohexenone and dimethylalumino trimethylsilylacetylide catalyzed by a tetrahedral complex prepared from $\mathrm{Ni}(\mathrm{II})$ acetylacetonate and bisoxazoline-type ligands (Scheme 28). Under the optimized conditions the alkynylation product was obtained with $86 \%$ yield and $82-88 \%$ ee. No other examples were reported. Remarkably, the use of $\mathrm{Ni}(\mathrm{I})$, as described in a non-enantioselective alkynylation by Schwartz, ${ }^{10 b}$ led to lower yields and enantioselectivities compared with $\mathrm{Ni}(\mathrm{II})$.

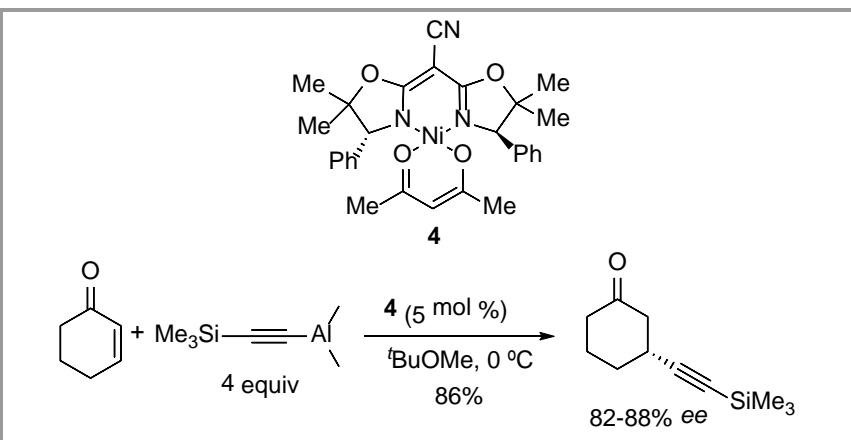

Scheme 28 Enantioselective conjugate addition of alkynylalanes to 2cyclohexenone catalyzed by a Ni(II)-bisoxazoline-acetylacetonate complex

The authors proposed the mechanistic pathway described in Scheme 29, which involved the carbometalation of the $\alpha, \beta$ enone by an alkynyl-nickel intermediate as a key step.
In a later work, the same group reported the conjugate alkynylation of cyclic $\alpha, \beta$-enones using a different $\mathrm{Ni}(\mathrm{II})$ complex with a chiral bis-phosphine ligand. This complex catalyzed the addition of diisobutyl(phenylethynyl)aluminum, prepared from phenylethynyllithium and diisobutylaluminum chloride, to several cyclic enones. Although 2-cyclopentenone appeared to be unsuitable for the reaction, larger cyclic enones underwent conjugate additions of the alkynyl-diisobutylaluminum reagent to form the corresponding ketones with $85-90 \%$ ee (Scheme 30). The addition of diisobutyl(trimethylsilylethynyl)-aluminum to 2-cyclohexenone was also reported, with results similar to those obtained previously with the Ni(II)-bisoxazoline complex. ${ }^{39}$

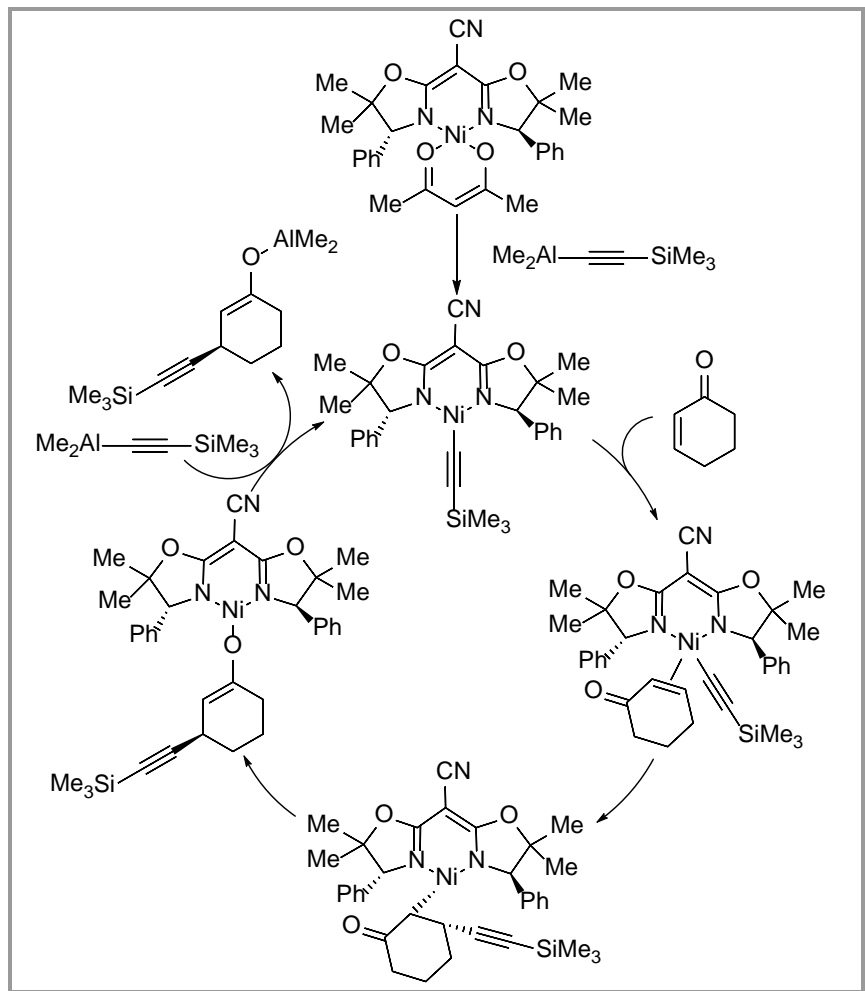

Scheme 29 Mechanistic pathway for the $\mathrm{Ni}(I I)$-catalyzed conjugate alkynylation of 2-cyclohexenone.

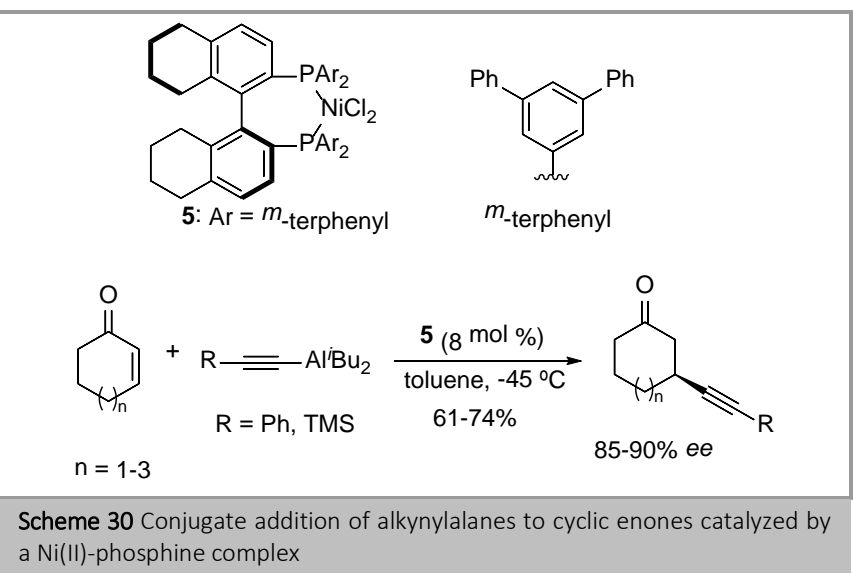

\subsubsection{Organocatalytic addition of alkynylboranes}

In 2005, the group of Chong developed a catalytic version of his previous reaction with chiral alkynylboronates. ${ }^{35}$ In this new work, the authors achieved the conjugate alkynylation of enones 
with $B$-(1-alkynyl)diisopropylboronates in the presence of a catalytic amount of a 3,3'-disubstituted BINOL. The best results were obtained with 3,3'-diiodo-1,1'-binaphthol (3,3'- $\mathrm{I}_{2}$-BINOL) that provided the alkynylation products with good yields and high enantiomeric excesses for a number of enones bearing $\beta$ alkyl or aryl substituents, and alkynes substituted with aryl or alkyl groups (Scheme 31). ${ }^{40}$

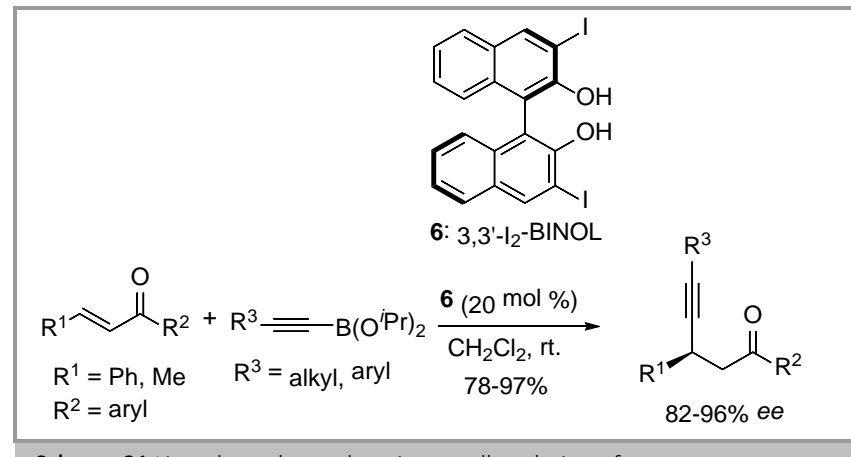

Scheme 31 Ligand-accelerated conjugate alkynylation of enones

The catalytic reaction provided similar enantioselectivity to those obtained with the reaction performed with stoichiometric amounts of chiral boronates, regardless of the catalytic load. The results suggest a ligand-accelerated asymmetric process since the diisopropylboronate reagent was unreactive. Activation by simple aliphatic diols such as ethylene glycol and pinacol proved not effective, while diisopropyl tartrate catalyzed the reaction but gave racemic products, and other bidentate ligands, such as $N$-tosyl aminoacids, also catalyzed the reaction but with low ee.

The authors proposed the catalytic cycle depicted in Scheme 32, in which liberation of the chiral binaphthol occurs by ligand exchange/disproportionation with the achiral boronate to regenerate the reactive species along with the reaction product. Theoretical studies performed by Goodman confirmed this mechanism and attribute the higher reactivity of the chiral boronate intermediate to the lower delocalization of the oxygen electron pairs toward the boron atom, which increases its Lewis acid character to activate the enone, compared with the diisopropylboronate. These calculations also showed the importance of steric interactions of the substituent at the 3 and 3' positions of the catalyst as responsible for the facial enantiodiscrimination. ${ }^{41}$

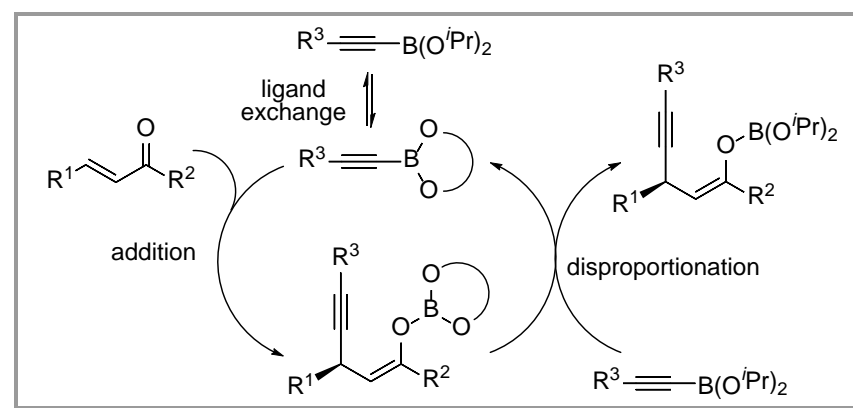

Scheme 32 Catalytic cycle for the BINOL-catalyzed alkynylation of enones with alkynylboronates

\subsubsection{Copper catalyzed conjugate alkynylations}

The first copper catalyzed enantioselective conjugate addition of terminal alkynes to unsaturated carbonyl compounds was reported by Carreira in $2005 .{ }^{42}$ Under similar conditions to those used by the same group in the previous nonenantioselective reaction, ${ }^{30}$ phenylacetylene was reacted with doubly activated alkenes derived from Meldrum's acid in the presence of copper acetate, sodium ascorbate and PINAP as chiral ligand (Scheme 33).

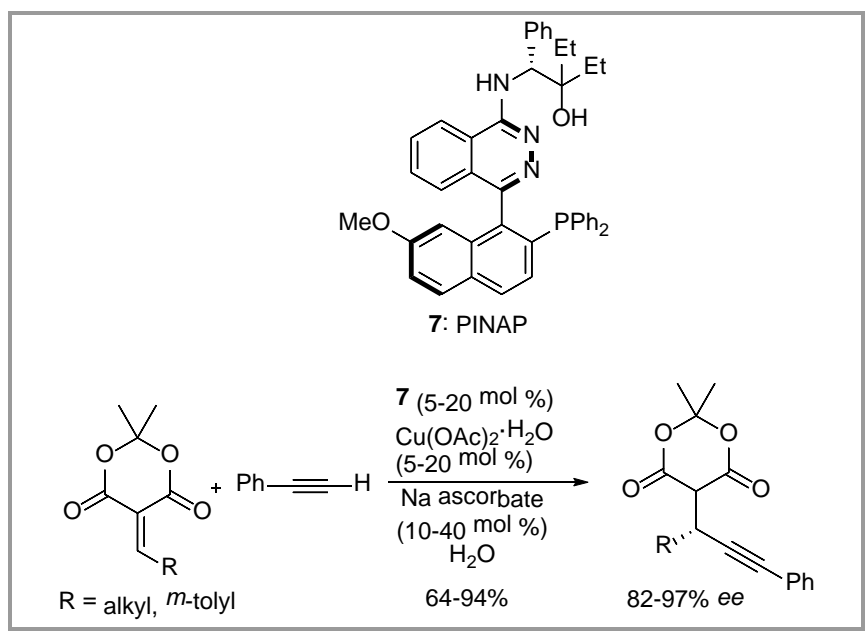

Scheme 33 Enantioselective conjugate alkynylation of Meldrum's acid derivatives catalyzed by $\mathrm{Cu}(\mathrm{I})$-PINAP

The reaction is carried out in heterogeneous phase and efficient stirring is essential for good conversions. According to the authors, formation of the reactive copper alkynylides takes place in the water phase while the conjugate addition occurs in the organic phase, namely the alkyne. The presence of sodium ascorbate avoids re-oxidation of $\mathrm{Cu}(\mathrm{I})$ to $\mathrm{Cu}(\mathrm{II})$, and the reaction can be carried out to give the expected products with good yields and enantiomeric excesses, without the need of inert atmosphere. Aliphatic alkynes also reacted under these conditions although with lower yield and enantioselectivity. In a later work, the authors noticed a positive non-linear effect when using a diastereomeric mixture of PINAP ligands, and found that a 60:40 mixture was enough to reach high enantioselectivity in the conjugate addition of phenylacetylene. ${ }^{43}$

Recently, Aponiack developed new atropisomeric $P, N$-ligands, StackPhos, that were applied as substitutive for the PINAP ligand in the alkynylation of Meldrum's acid derivatives under Carreira's conditions. A variety of functionalized alkynes were successfully reacted. The authors demonstrated the applicability of the reaction with the synthesis of the preclinical agent OPC 51803 (Scheme 34). ${ }^{44}$

In 2010, Shibasaki described the enantioselective conjugate addition of terminal alkynes to $\alpha, \beta$-unsaturated thioamides under proton transfer conditions in the presence of a copper (I)bis-phosphine complex as soft Lewis acid catalyst. ${ }^{45}$ The authors envisioned that the use of a soft Lewis acid would enable the simultaneous activation of the soft Lewis basic thioamides and terminal alkynes via a soft-soft interaction enabling high chemoselectivity and efficient catalytic turnover. On the other hand, the chiral copper acetylides were catalytically generated by a soft Lewis acid/hard Brønsted base/hard Lewis base cooperative catalyst prepared from $\left[\mathrm{Cu}\left(\mathrm{CH}_{3} \mathrm{CN}\right)_{4}\right] \mathrm{PF}_{6}$, the MeOBIPHEP bis-phosphine ligand as chiral inducer (BIPHEP $=2,2$ 'biphenylphosphine), $\mathrm{Li}\left(\mathrm{OC}_{6} \mathrm{H}_{4}-p-\mathrm{OMe}\right)$ as hard Brønsted base. 
The combined use of bisphosphine oxide as a hard Lewis base that coordinates to lithium through a hard-hard interaction enhanced the Brønsted basicity of $\mathrm{Li}\left(\mathrm{OC}_{6} \mathrm{H}_{4}-p-\mathrm{OMe}\right)$, allowing for the completion of the reaction with a low catalytic load. Under these conditions, phenylacetylene and also 1cyclohexenylacetylene reacted with different unsaturated thioamides to give the expected alkynylated products with good yields and enantioselectivity (Scheme 35).

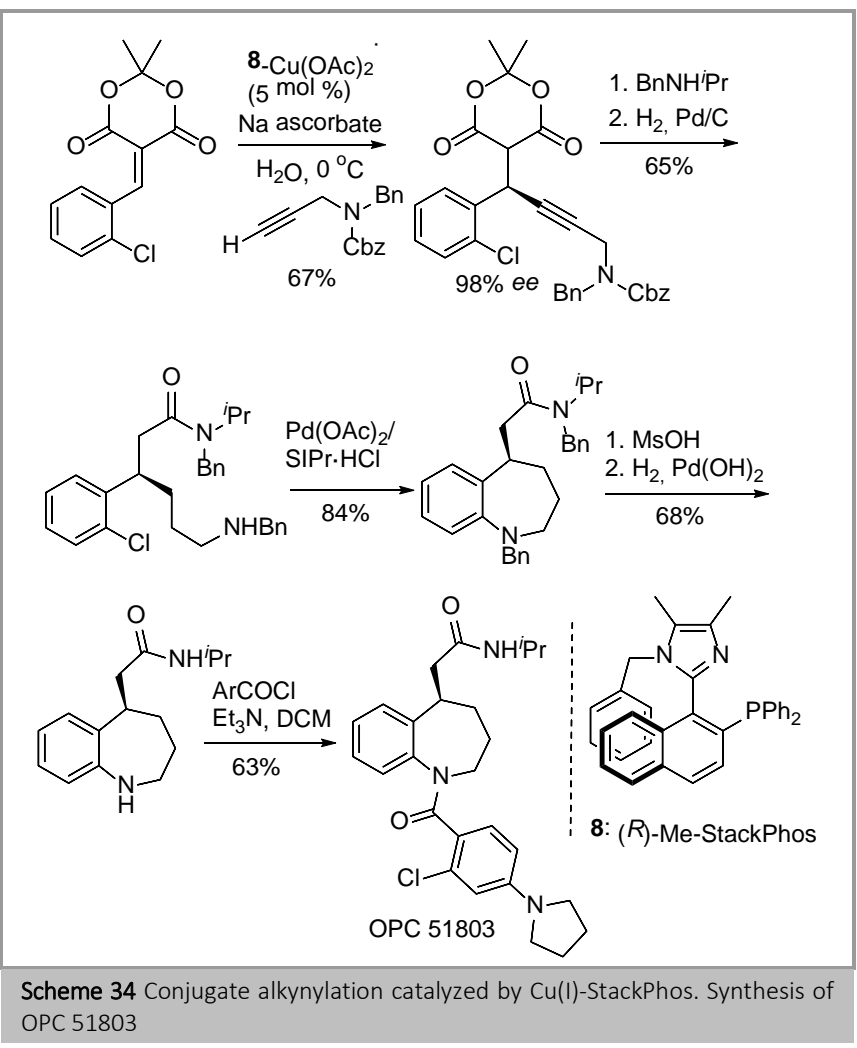

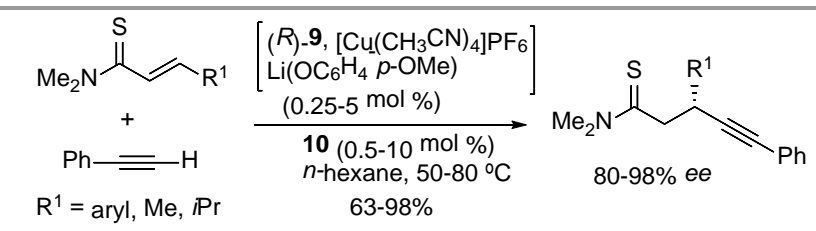

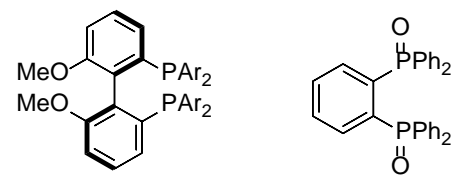

(R)-9: $\mathrm{Ar}=3,5-{ }^{i} \mathrm{Pr}_{2}-4-\mathrm{Me}_{2} \mathrm{~N}-\mathrm{C}_{6} \mathrm{H}_{2}$

10

Scheme 35 Enantioselective conjugate addition of phenylacetylene to $\alpha, \beta$ unsaturated thioamides

However, saturated aliphatic terminal alkynes afforded moderate enantioselectivity under these conditions. To overcome this drawback, the catalytic system was reinforced with the use of a copper salt with a chiral counter anion, prepared from mesitylcopper and a chiral phosphoric acid, generating a catalyst armed with a chiral bulky phosphate anion in proximity to the $\mathrm{Cu}$ cation. The chiral phosphate was also expected to act as a hard Lewis base, similar to the phosphine oxide in the previous system, to enhance the reaction rate. With this catalyst, high enantioselectivity was observed in the addition of a series of aliphatic alkynes (Scheme 36). Matching of the stereochemistry of the bis-phosphine ligand and the phosphoric acid is crucial, since no reaction was observed when the $S$ phosphoric acid was used instead of the $R$ enatiomer.

After a series of control experiments the authors concluded that the transient thioamide enolate that results after nucleophilic addition of the copper alkynylide functions as a Brønsted base that directly deprotonates a new terminal alkyne to release the product with concomitant regeneration of the copper alkynylide that engages in a new catalytic cycle (Scheme 37).

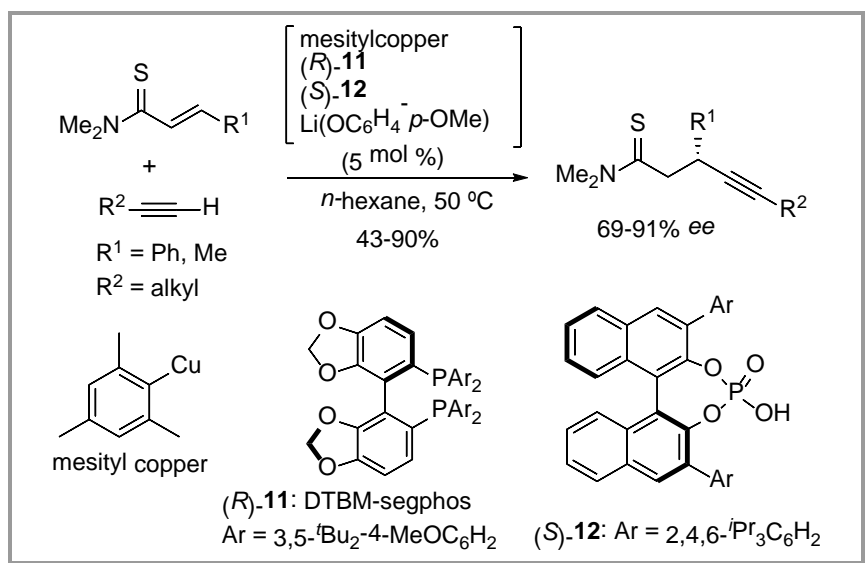

Scheme 36 Enantioselective conjugate addition of aliphatic alkynes to $\alpha, \beta$ unsaturated thioamides

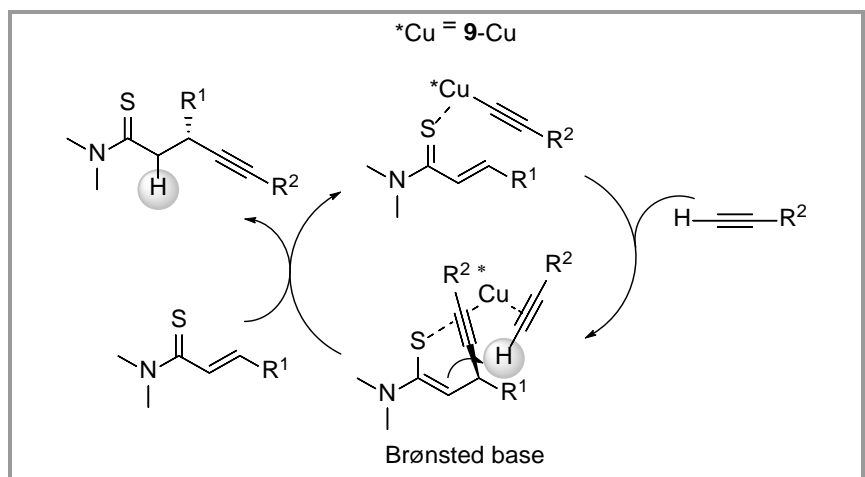

Scheme 37 Catalytic cycle for the $\mathrm{Cu}(\mathrm{I})$-catalyzed conjugate alkynylation of unsaturated thioamides

The authors reported different transformations of the thioamide moiety into carboxylic acid derivatives (Scheme 38).

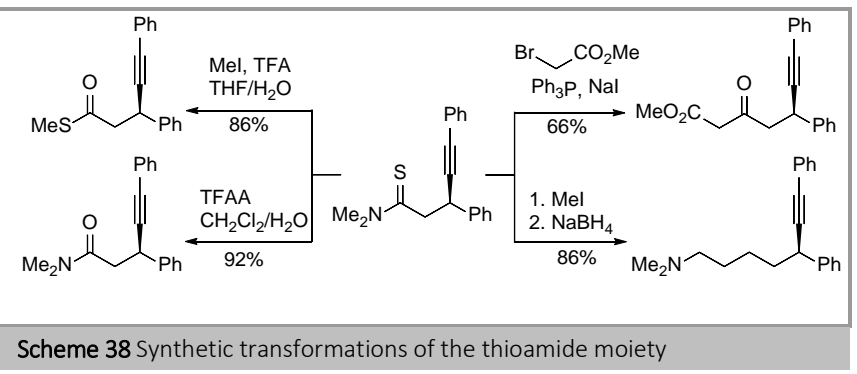

The utility of this reaction was demonstrated by the authors with another synthesis of the GPR40 agonist AMG 837 (Scheme 39). ${ }^{46}$ Application of the original conditions for the enantioselective conjugate alkynylation in the synthesis of this compound was not effective probably due to the high steric 
demand in the required electrophile. Substitution of the lithium $p$-methoxyphenoxide by a stronger base such as LiHMDS permitted to achieve the alkynylation with $83 \%$ yield and $91 \%$ $e e$.

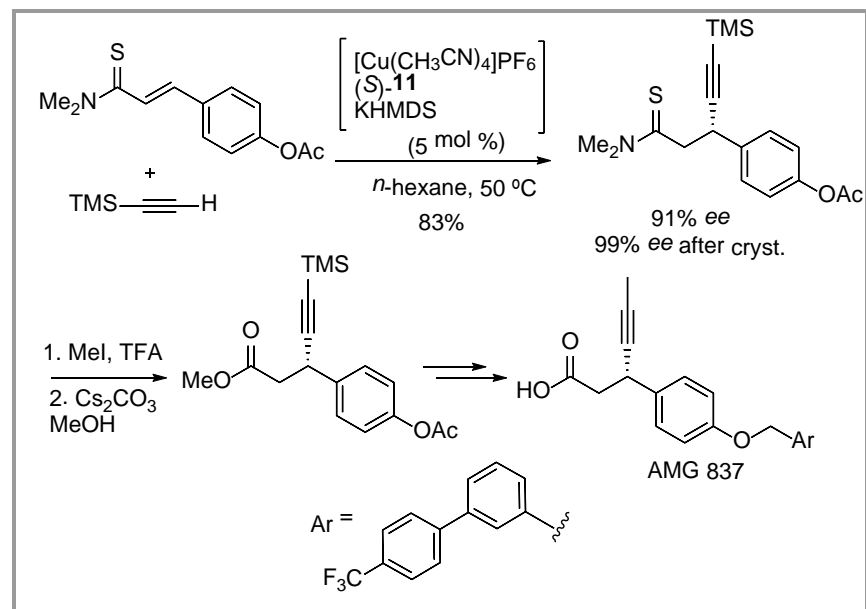

Scheme 39 Application of the Cu-catalyzed alkynylation of unsaturated thioamides to the synthesis of AMG 837

Since the success of the copper-catalyzed conjugate alkynylation was shown largely dependent on the electrophile features, Pedro and Blay introduced 1,1-difluoro-1-(phenylsulfonyl)-3en-2-ones as substrates for this reaction. It was envisioned that the presence of two strong electronegative fluorine atoms and a sulfone electron-withdrawing group next to the carbonyl group would largely enhance the electrophilicity of the double bond, thus overcoming the low reactivity of copper alkynylides. Accordingly, a number of these substrates having aromatic substituents at the $\beta$-position reacted smoothly with phenylacetylene derivatives, 3-thienylacetylene and cyclopropylacetylene in the presence of a copper complex generated in situ from $\left[\mathrm{Cu}\left(\mathrm{CH}_{3} \mathrm{CN}\right)_{4}\right] \mathrm{BF}_{4}$ and the MeO-BIPHEP ligand, and triethylamine to give the corresponding products with good yields and excellent enantioselectivities (from 92 to 99\% ee). ${ }^{47}$ Unfortunately, neither $\beta$-alkyl-substituted enones nor aliphatic alkynes were reactive under these conditions (Scheme 40).

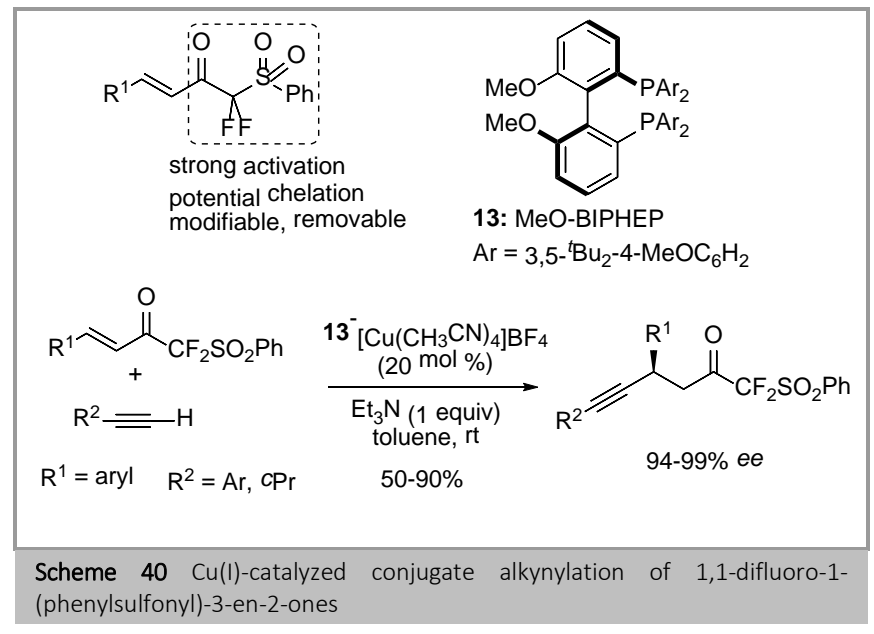

The difluoro(phenylsulfonyl)methyl moiety could be transformed into different fluorine-containing groups. Thus reductive elimination upon treatment with $\mathrm{Mg}$ and TMSCl gave an enolate ether that could be converted into the difluoro- or trifluoromethyl ketone after quenching with aqueous $\mathrm{HF}$ or Selectfluor, respectively. Furthermore, the difluoro(phenylsulfonyl)methyl moiety could be replaced by a methoxy group or an amine to give the corresponding $\beta$ alkynylated ester or amide (Scheme 41).

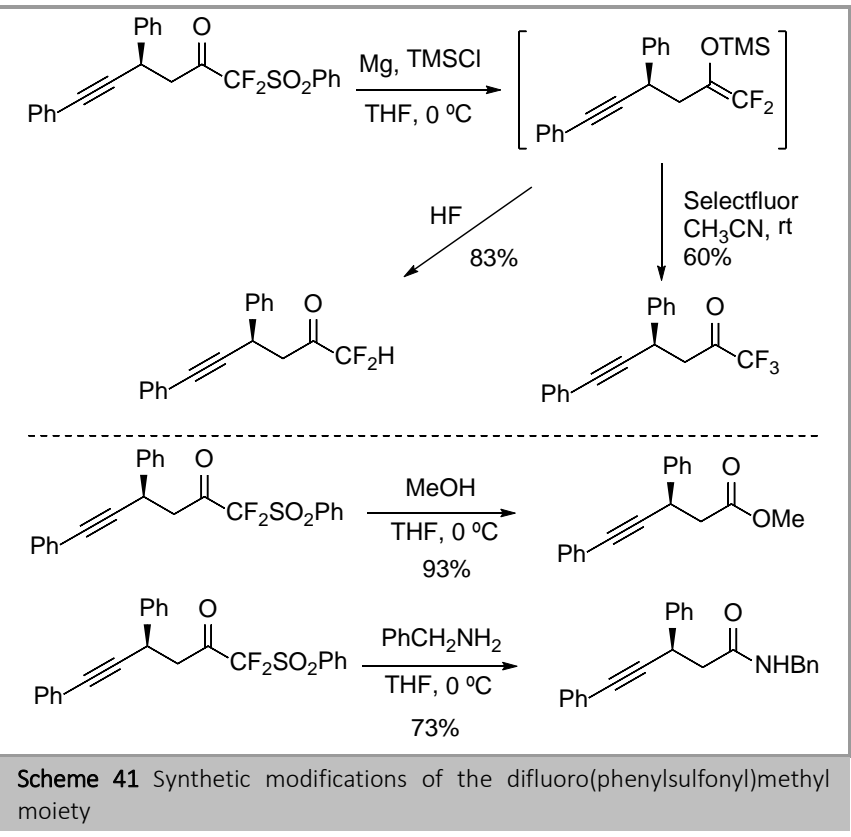

$\alpha, \beta$-Unsaturated 1,1,1-trifluoromethyl ketones could also be alkynylated using the same catalyst, although in this case it was necessary to reduce the catalyst load as well as the amounts of alkyne and base to avoid the formation of the 1,2- and 1,4double alkynylation product. Under the modified conditions the $\beta$-alkynylated 1,1,1-trifluoromethyl ketones were obtained with good yields and excellent enantiomeric excesses. Again, aryl and heteroaryl but not alkyl substituents on the $\beta$-position were tolerated. Remarkably, besides substituted phenylacetylenes, phenyl-1-butyne was also reactive, although in this case a somehow larger catalyst load was required and the enantioselectivity obtained was slightly lower than that observed with aromatic alkynes (Scheme 42). ${ }^{48}$

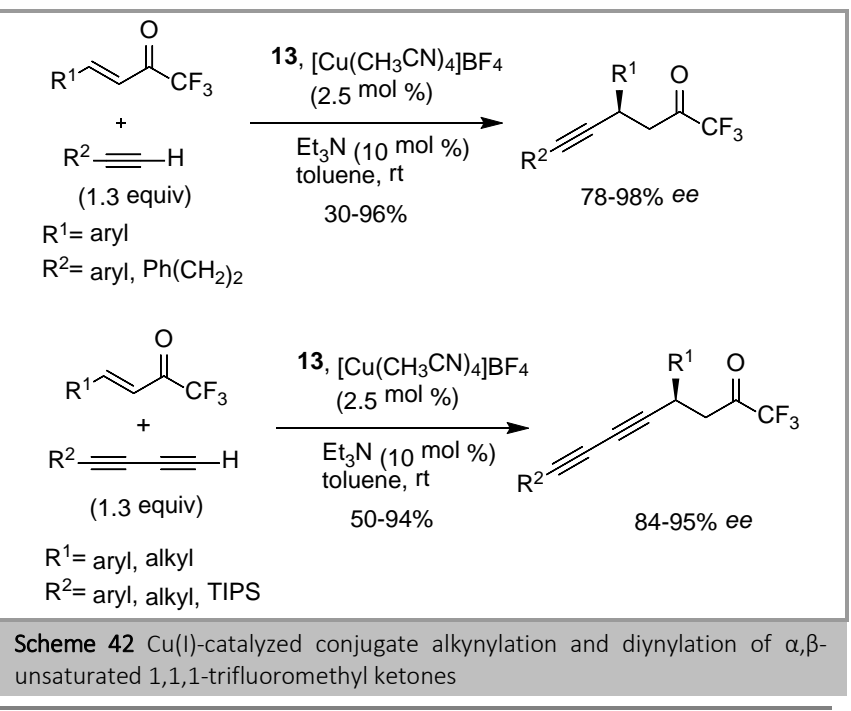


The same catalytic system allowed the conjugate addition of 1,3diynes to $\alpha, \beta$-unsaturated 1,1,1-trifluoromethyl ketones with broad substrate and alkyne scope. Thus, the reaction worked not only with enones bearing aromatic $(e e>90 \%)$ but also alkyl (ee $>80 \%$ ) substituents on the $\beta$-position. Furthermore, 1,3diynes having aryl, alkyl and silyl groups at position 4 were suitable nucleophiles providing the reaction products with good yields and excellent enantioselectivity (Scheme 42).

A plausible catalytic cycle for the conjugate alkynylation of trifluoromethyl enones was proposed by the authors (Scheme 43). The reaction between the cationic complex $\left[\mathrm{Cu}\left(\mathrm{CH}_{3} \mathrm{CN}\right)_{4}\right] \mathrm{BF}_{4}$ and the MeO-BIPHEP ligand would form the catalytic complex, which upon $\pi$-complexation to the $\mathrm{C}$-C triple bond can enhance the acidity of the terminal proton of the alkyne in such a way that it can be deprotonated by triethylamine, forming the corresponding chiral copper acetylide which may be in equilibrium with a dimer. The copper-acetylide species then would form a $\pi$-complex with the double bond of the enone bringing both reaction substrates to proximity. Transfer of the alkyne to the $\beta$-carbon of the enone, the enantioselectivity-determining step, probably through a $\mathrm{Cu}(\mathrm{III})$ intermediate would lead to a copper enolate, which after protonation by triethylamonium would release the reaction product with regeneration of the catalyst.

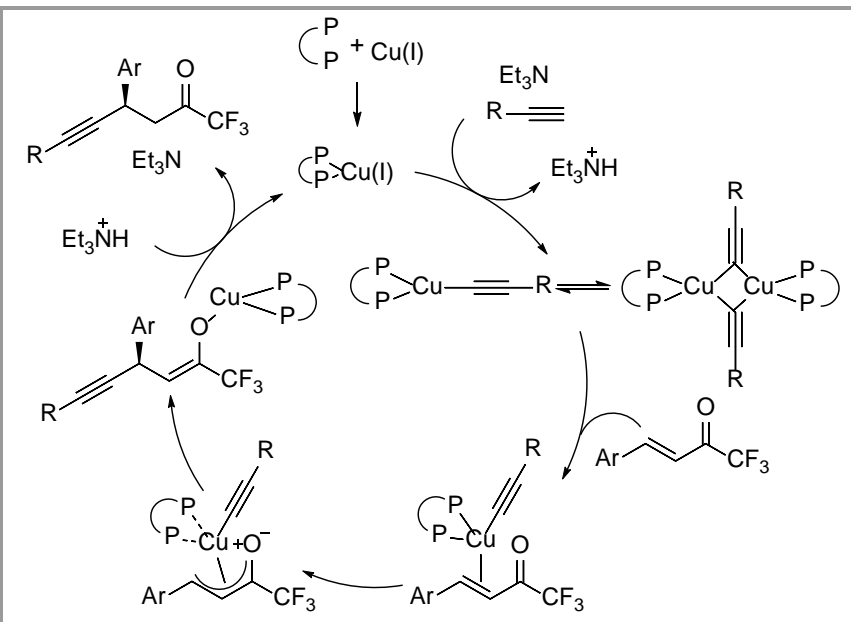

Scheme 43 Proposed mechanism for the $\mathrm{Cu}(\mathrm{I})$-catalyzed conjugate alkynylation of $\alpha, \beta$-unsaturated trifluoromethyl ketones

A rationale of the observed stereochemistry was proposed following a model in which the axial-chirality information of the biphenyl backbone is transferred through the P-aryl rings, which define four differentiated quadrants in the space around the metal center, two of them open for approach of substrates and reagents. Formation of the stereogenic center takes place through a tetracoordinated $\mathrm{Cu}(\mathrm{I})$ species in which the $R e$-face of the double bond is exposed to attack by the alkyne while the $\mathrm{Si}$ face is shielded by one aryl ring of the $(R)$-MeO-BIPHEP (Figure1).

Finally, our group has also reported the first enantioselective conjugate alkynylation of $\beta$-trifluoromethyl $\alpha, \beta$-enones using terminal alkynes and a taniaphos-Cu(I) complex as catalyst. ${ }^{49}$ The reaction furnished ketones bearing a trifluoromethylated propargylic chiral center in the $\beta$-position. Good yields and enantiomeric excesses were obtained in the addition of phenylacetylene derivatives, 2-thiophenylethyne and 4-phenyl1-butyne to enones having aromatic groups attached to the carbonyl group, especially with 2-thienyl derivatives. Enones having a phenylethyl group attached to the ketone reacted with good enantiomeric excesses but moderated yield, while a ketone substituted with a $n$-butyl group was not reactive (Scheme 44).

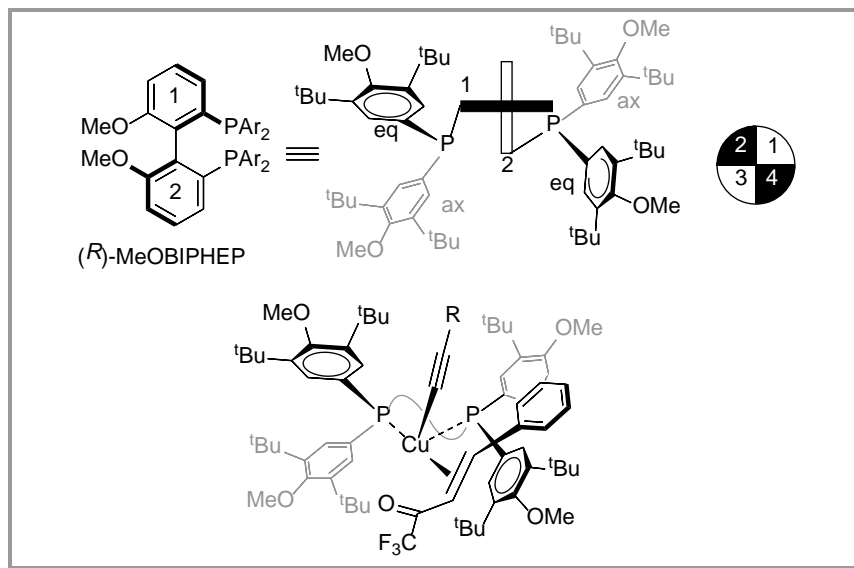

Figure 1 Structure of C2-symmetric biaryl phosphine ligands and stereochemical model for the enantioselective conjugate alkynylation ${ }^{48 b}$

$\mathrm{R}^{1=}$ aryl, 2-thienyl, $\mathrm{Ph}_{\left(\mathrm{CH}_{2}\right) 2}$
$\mathrm{R}^{2}=$ aryl, 3-thienyl, $\mathrm{Ph}_{\left(\mathrm{CH}_{2}\right) 2}$
Scheme 44 Catalytic asymmetric conjugate addition of terminal alkynes to $\beta$ -
trifluoromethyl $\alpha, \beta$-enones

The alkynyl trifluoromethyl ketones could be subjected to different cyclization reactions leading to heterocyclic compounds (Scheme 45).

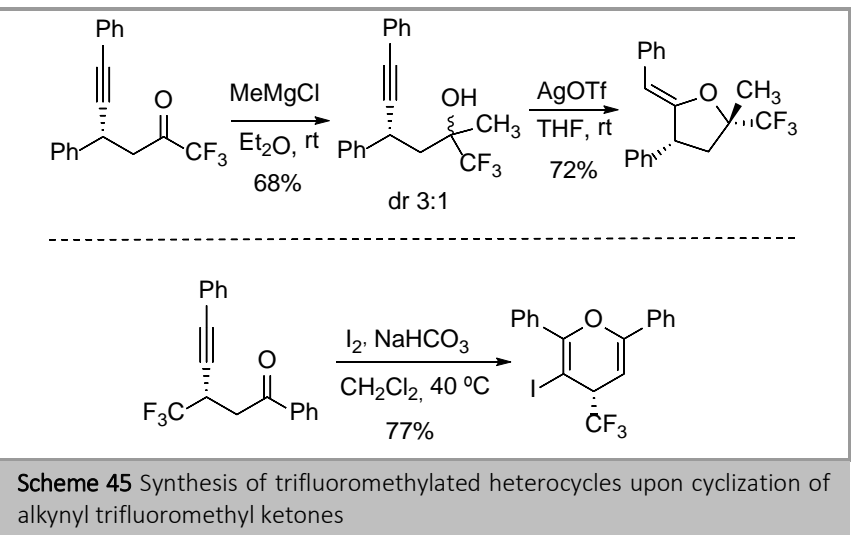

Thus, treatment of the $\beta$-alkynyl 1,1,1-trifluoroketones with methylmagnesium chloride followed by silver-catalyzed 
cyclization of the resulting alcohol gave dihydrofurans bearing a trifluoromethylated quaternary stereocenter. On the other hand, $\beta$-alkynyl- $\beta$-trifluoromethyl ketones gave dihydropyrans upon treatment with iodine in basic medium.

\subsubsection{Rhodium catalyzed conjugate alkynylations}

In 2007 Nishimura and Hayashi found a new method of introducing alkynyl groups to the $\beta$-position of $\alpha, \beta$-unsaturated ketones with high enantioselectivity. The procedure involved the asymmetric 1,3-migration of alkynyl groups from 1 to 3 in alkynyl alkenyl carbinols, which could be obtained by addition of lithium tert-butyldimethylsilylacetylide to enones (Scheme 46). ${ }^{50}$ Excellent yields and enantiomeric excesses were obtained for a number of carbinols having the $E$ configuration at the double bond using the $(R)$-BINAP-Rh(I) complex as catalyst. In the reaction of cyclic alcohol derived from indenone the enantioselectivity was moderated under standard conditions, but the use of chiral diene ligand Ph-BOD (BOD = bicyclo[2.2.2]octane-2,5-diene) for the triisopropylsilyl (TIPS) analogue resulted in a great increase of enantioselectivity (Scheme 47).
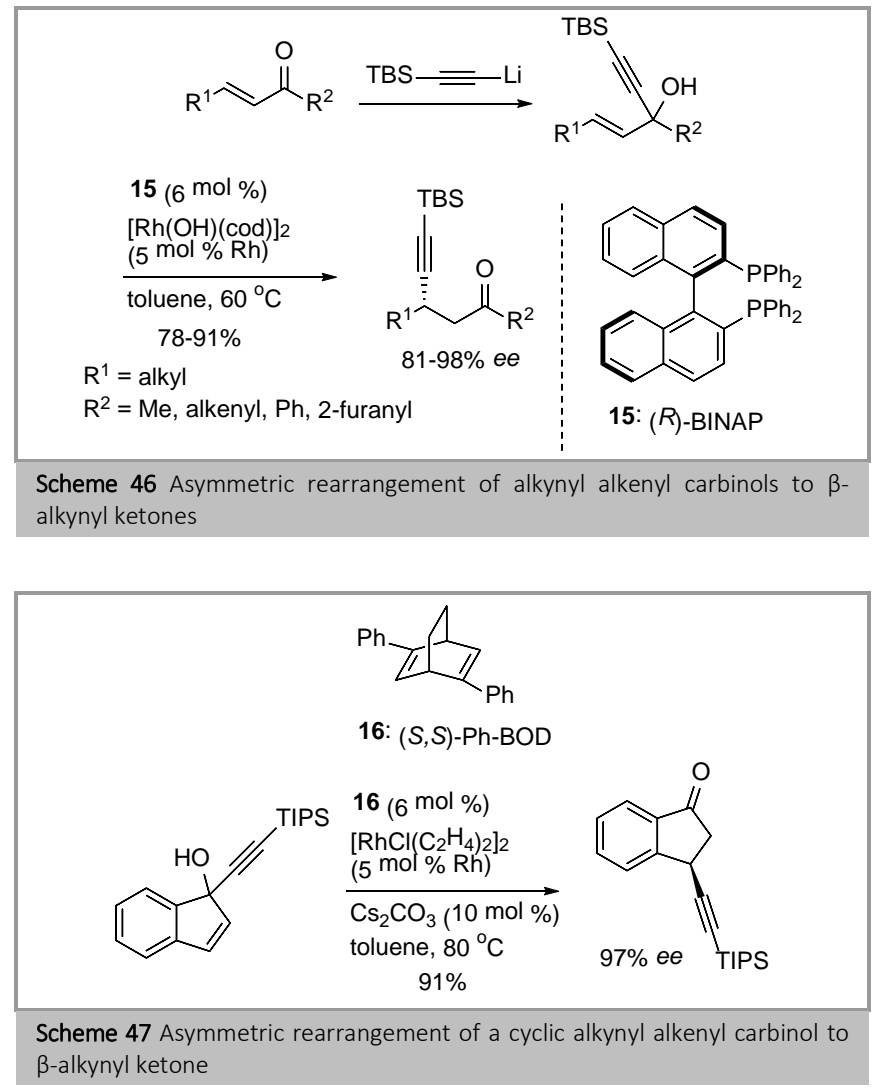

The rearrangement was catalyzed by a chiral $\mathrm{Rh}(\mathrm{I})$-diene complex and probably involved the $\beta$-alkynyl elimination from an alkoxyrhodium intermediate followed by the conjugate addition of the resulting alkynylrhodium species to the enone (Scheme 48).

One year later,51 the same group described the first enantioseletive conjugate alkynylation of enones catalyzed by $\mathrm{Rh}$, using triisopropylsilylacetylene and a complex generated from $\left[\mathrm{Rh}\left(\mu \text {-OAc) }\left(\mathrm{C}_{2} \mathrm{H}_{4}\right)_{2}\right]_{2}\right.$ and the $(R)$-DTBM-segphos ligand as catalyst (Scheme 49). Several $\beta$-alkyl substituted enones were alkynylated with good yields (54-99\%) and high enantiomeric excesses $(89-95 \% e e)$. The presence of bulky groups such as triisopropyl in the alkyne and 3,5-di-tert-butyl-4methoxyphenyl in the phosphine ligand was essential to suppress the dimerization of the alkyne, which was detrimental to the yield, especially with less reactive enones. The reaction was also applicable to 2-cyclopentenone derivatives, although in this case the yields were lower (Scheme 49). The same catalyst could be applied in the addition of triisopropylsilylacetylene to enals with excellent results. The reaction in 1,4-dioxane as described previously for enones gave the product of double alkynylation as the major product; nevertheless, 1,2alkynylation could be suppressed by using $\mathrm{MeOH}$ as the solvent. Other trialkylsilylacetylenes, phenyl acetylene or butyne gave the 1,4-addition products but with low ee. ${ }^{52}$

$$
\text { Scheme 48 Mechanism for the rearrangement of alkynyl alkenyl carbinols }
$$

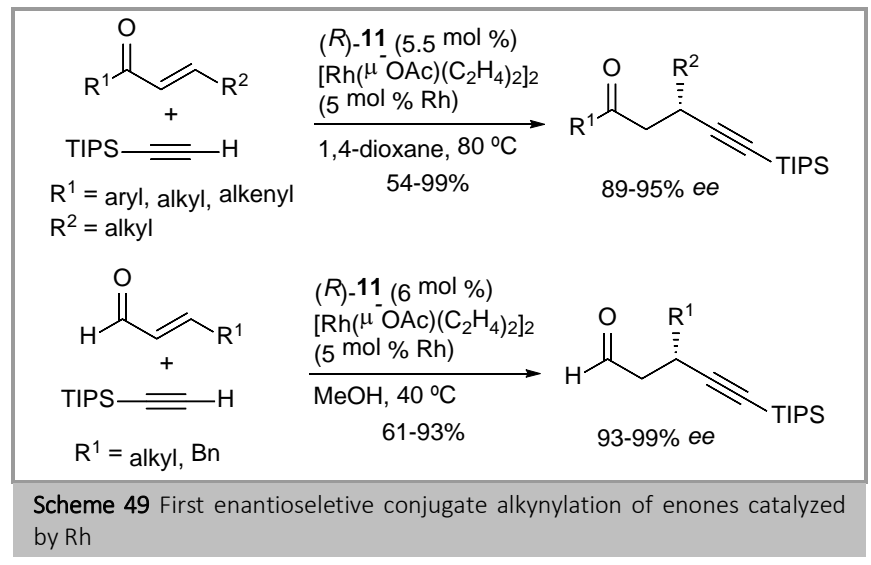

To explain the absolute stereochemistry of the alkynylated products, the authors proposed the stereochemical pathway shown in Figure 2, with the alkynylrhodium attacking the double bond of the enal which would be positioned to minimize the repulsion with one of the aromatic substituents of the phosphine.

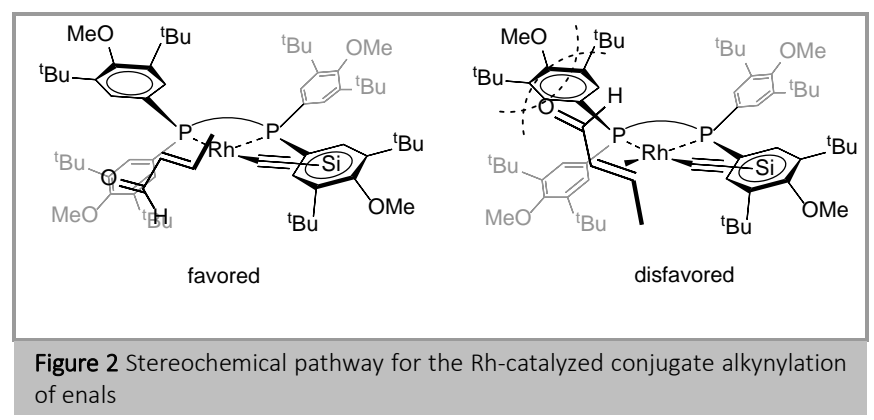


The synthetic value of the rhodium-catalyzed alkynylation in generating stereogenic centers has been demonstrated recently with the enantioselective synthesis of mandelalide $\mathrm{A}$ and aphanamol I (Scheme 50). ${ }^{53}$

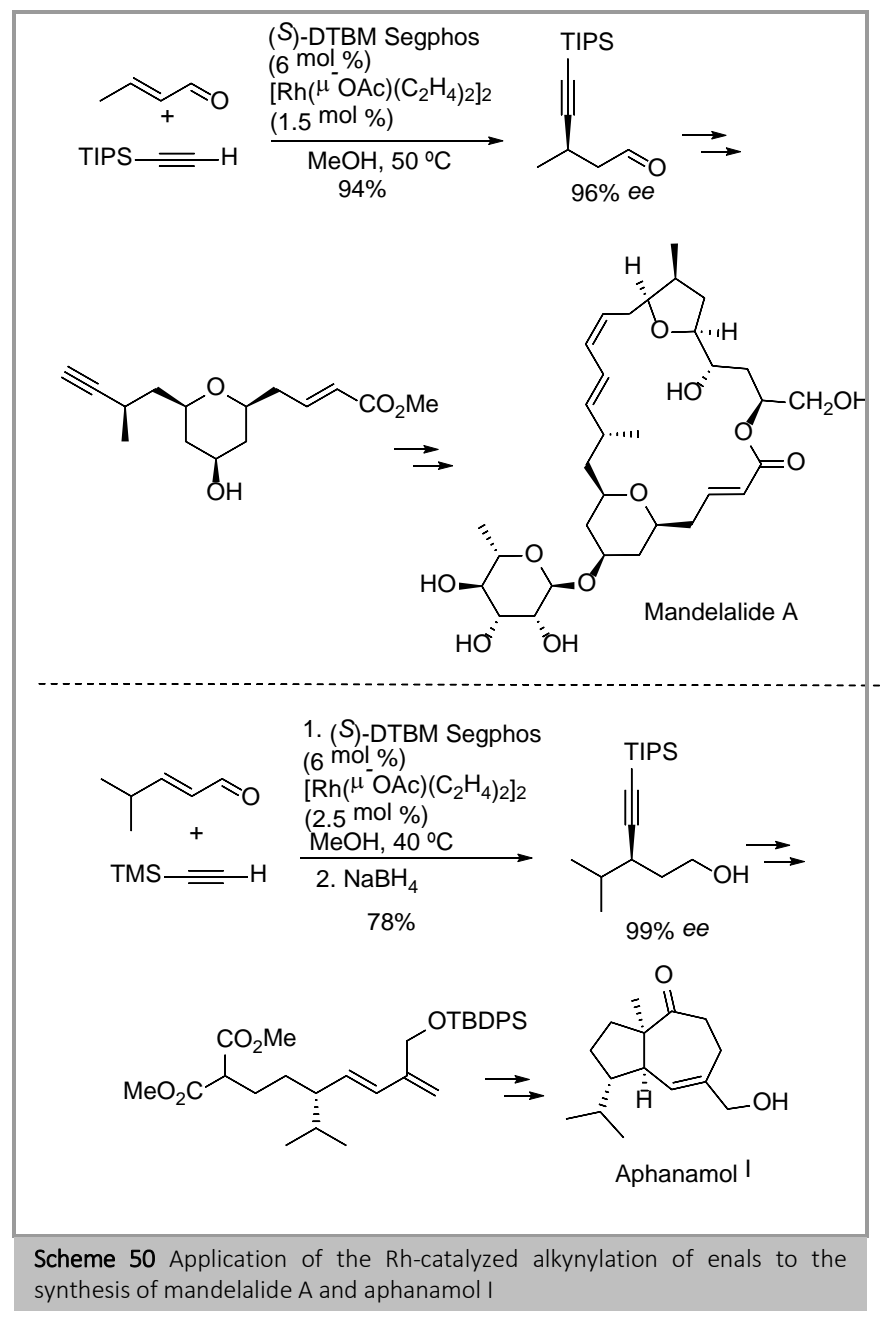

As a way of reducing alkyne dimerization in the alkynylation of less reactive enones, i. e. $\beta$-aryl enones, Nishimura and Hayashi employed alkynylsilanols as precursors of the $\mathrm{Rh}(\mathrm{I})$ alkynylides. By using different $\mathrm{Rh}(\mathrm{I})$ complexes these authors could achieve the alkynylation of different enones with good yields and enantioselectivity (Scheme 51). ${ }^{54}$

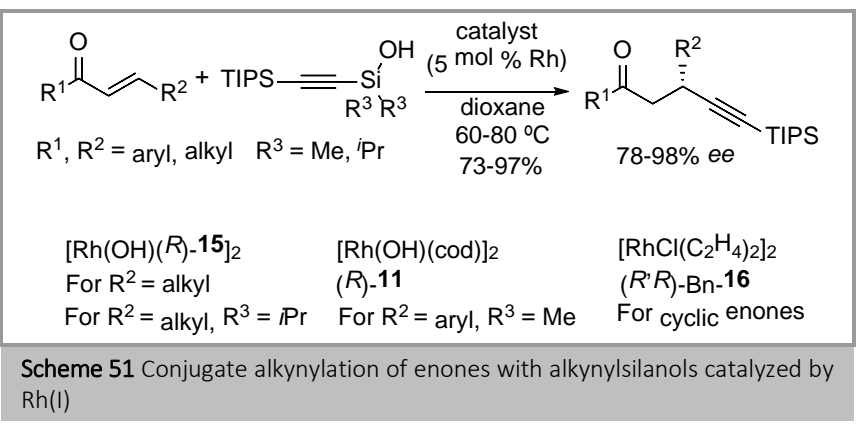

Although the authors developed conditions for the alkynylation of cyclic enones with the alkynylsilanols, the substrate scope was narrow and excess of alkynylating reagent was needed. To overcome this drawback they used diphenyl[(triisopropylsilyl)ethynyl]methanol as alkynylating reagent, which in combination with a complex of rhodium and a diene ligand bearing a ferrocenyl group, allowed the enantioselective alkynylation of cyclic enones, unsaturated lactones, and unsaturated lactams with excellent results (Scheme 52). ${ }^{55}$ Diphenyl[(triisopropylsilyl)ethynyl]methanol was also used by Lu and Dou to carry out the conjugate alkynylation of $\beta, \gamma$-unsaturated- $\alpha$-ketoesters. A Rh(I) complex with a BINAP complex was the catalyst for this reaction (Scheme $52) .56$

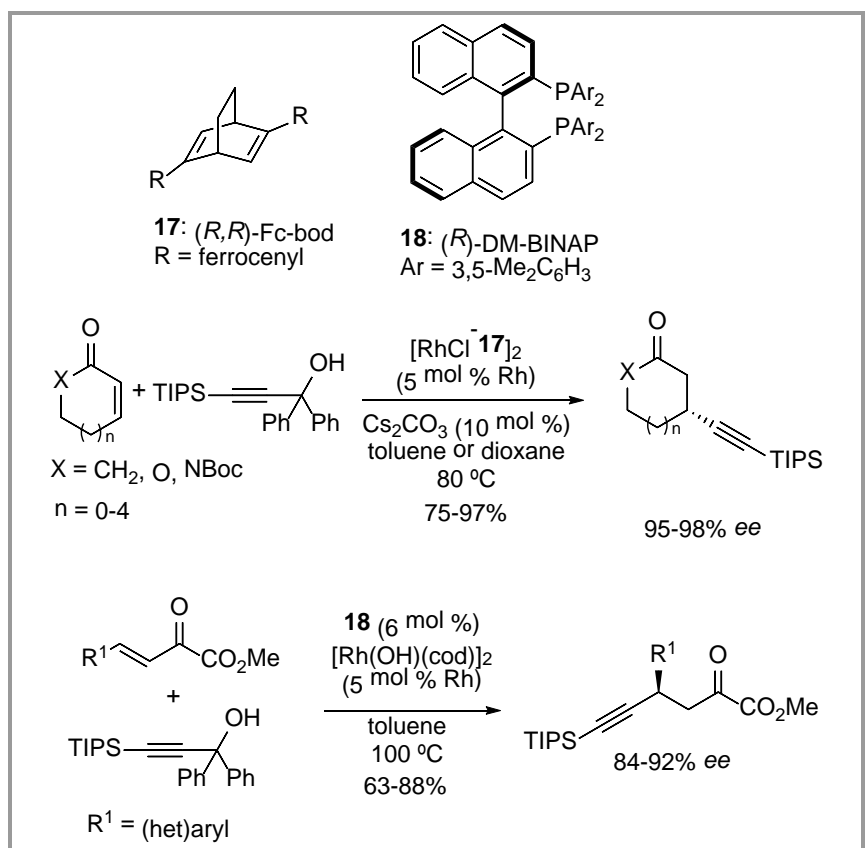

Scheme 52 Conjugate alkynylation of cyclic unsaturated carbonyl compounds and unsaturated keto esters with diphenyl[(triisopropylsilyl)ethynyl]methanol

Very recently, Lautens has used this reaction in an asymmetric conjugate alkynylation/aldol cyclization cascade leading to cyclic $\alpha$-propargyl- $\beta$-hydroxyketones, with simultaneous formation of a $\mathrm{C}(\mathrm{sp})-\mathrm{C}\left(\mathrm{sp}^{3}\right)$ bond, a $\mathrm{C}\left(\mathrm{sp}^{3}\right)-\mathrm{C}\left(\mathrm{sp}^{3}\right)$ bond and three new contiguous stereocenters. The reaction was achieved with excellent enantio- and diastereoselectivities using BINAP as the ligand in combination with $\left[\mathrm{Rh}(\mathrm{coe})_{2} \mathrm{Cl}_{2}\right.$ (coe = ciclooctene). While the reaction allowed variation at the diketone substrate, steric bulk flanking the propargylic carbon was required to avoid extensive homodimerization of the alkyne (Scheme 53). ${ }^{57}$

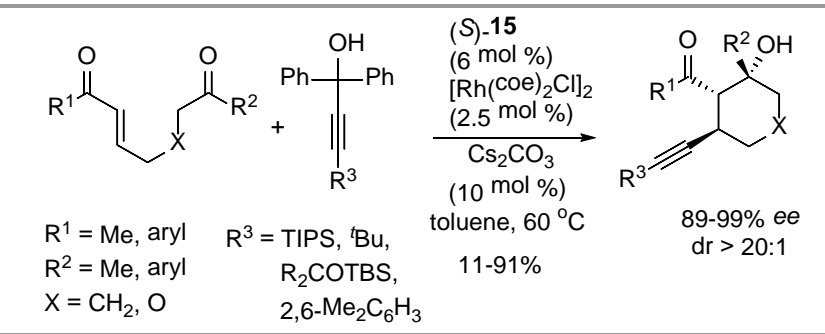

Scheme 53 Synthesis of $\alpha$-propargyl- $\beta$-hydroxyketones via an asymmetric conjugate alkynylation/aldol cyclization cascade

On the other hand, Fillion and Zorzitto have carried out the alkynylation of Meldrum's acid derivatives catalyzed by a $\mathrm{Rh}(\mathrm{I})$ complex with a chiral bis-phosphine ligand. The use of these 
highly electrophilic acceptors was envisioned as an alternative to the use of sterically shielded acetylenes to avoid competing terminal alkyne dimerization (Scheme 54). The authors performed the nucleophilic addition of trimethylsilylacetylene to a number of substrates with good yields and enantiomeric excesses, except for $N$-phenylamides. However, alkenes bearing 2 -methyl- or 4-methoxy- phenyl groups at the $\beta$-position were not suitable substrates in this reaction. ${ }^{58}$

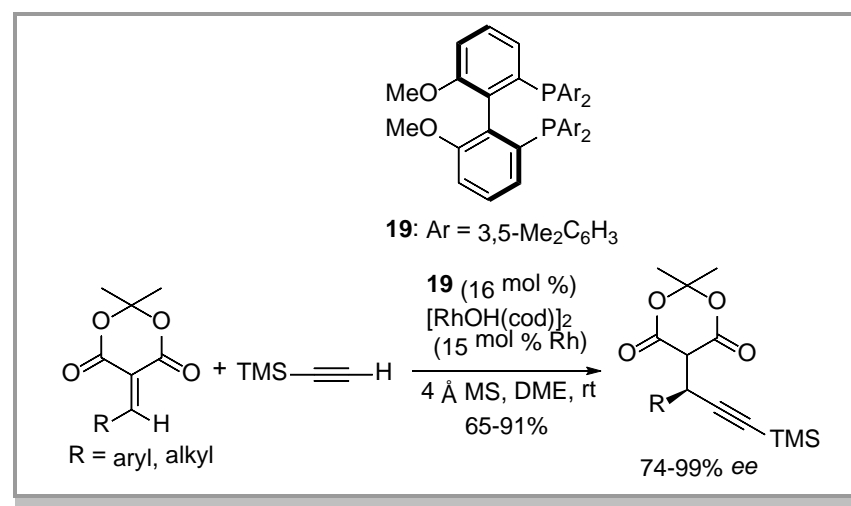

Scheme 54 Alkynylation of Meldrum's acid derivatives with trimethylsilylacetylene

\subsubsection{Cobalt catalyzed conjugate alkynylations}

Nishimura and Hayashi demonstrated in 2011 the ability of $\mathrm{Co}(\mathrm{I})$ to catalyze the addition of triisopropylsilylacetylene to $\alpha, \beta$-unsaturated ketones. ${ }^{59}$ The reaction is carried out with a $\mathrm{Co}(\mathrm{OAc})_{2} /$ bis-phosphine/Zn system where $\mathrm{Co}(\mathrm{II})$ is reduced to $\mathrm{Co}(\mathrm{I})$ by the zinc metal. Although most of the study was carried out with achiral phosphines, the use of $(S, S)$-BDPP allowed the enantioselective alkynylation of several enones with good yields and moderate enantioselectivity (Scheme 55).

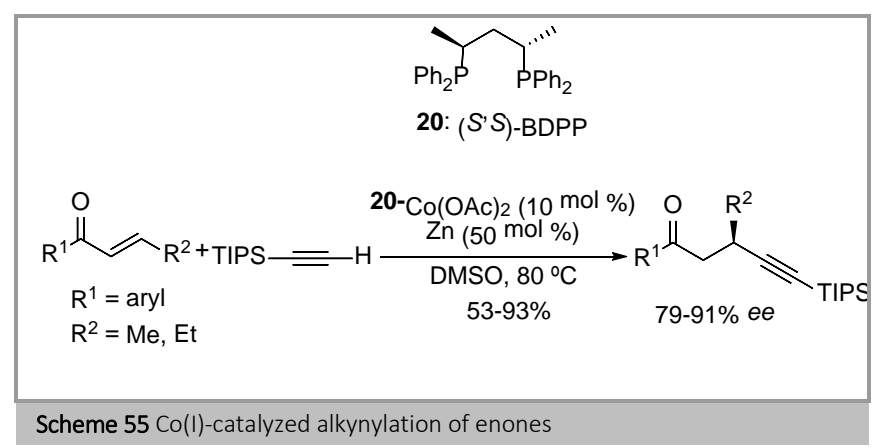

\subsubsection{Zinc mediated conjugate alkynylations}

In 2012, Blay and Pedro developed the first zinc mediated conjugate alkynylation requiring sub-stoichiometric amounts of chiral material, using doubly activated arylidene-1,3-diketones as electrophiles. The reacting nucleophile was generated by heating a terminal alkyne and $\mathrm{Et}_{2} \mathrm{Zn}$ in the presence of a catalytic amount of $(R)$-VANOL at $70{ }^{\circ} \mathrm{C}$, followed by addition of the electrophile at room temperature. The use of nitroethane as co-solvent was crucial to obtain good results. Arylidene-1,3diketones reacted with aromatic alkynes to give the conjugate alkynylation products with fair to good yields and good enantiomeric excesses (80-93\%). Alkylidene diketones were less reactive and gave lower enantiomeric excess, and similarly occurred with aliphatic alkynes (Scheme 56). ${ }^{60}$
On the other hand, the use of a novel bis-hydroxyamide ligand derived from 2-amino-2-(1-naphthylmethyl)-1,1diphenylethanol and isophthalic acid allowed the conjugate alkynylation of 3-tert-butoxycarbonylcoumarins with the system alkyne/Et $2 \mathrm{Zn}$ to give dihydrocoumarins with a propargylic stereogenic center at position 4. Phenylacetylene derivatives reacted with substituted coumarins with good yields and fair to good enantioselectivity, the best results being obtained with 8-substituted coumarins. 4-Phenyl-1-butyne could be also used but lower yields and enantiomeric excesses were obtained (Scheme 57). ${ }^{61}$

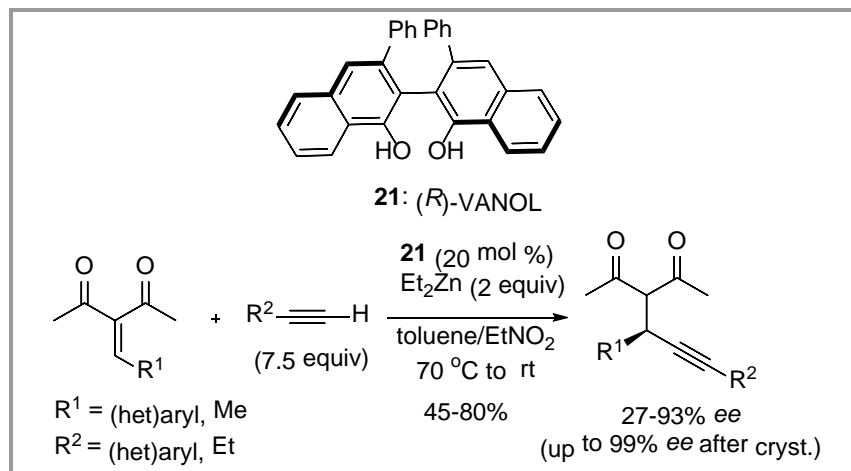

Scheme 56 Enantioselective zinc-mediated conjugate addition of terminal alkynes to arylidene 1,3-diketones

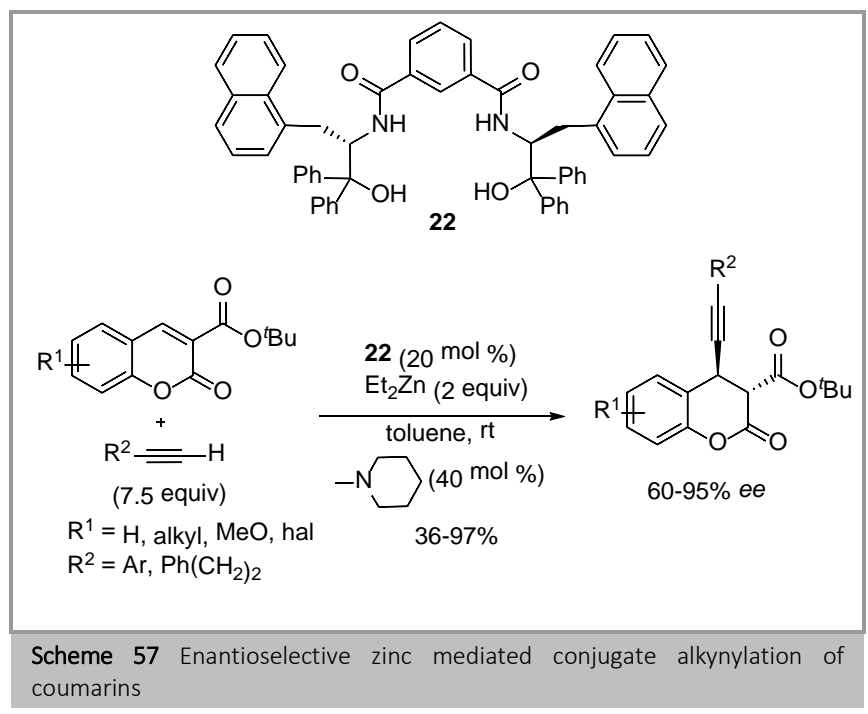

The tert-butoxycarbonyl, required for the reaction, could be removed under acidic conditions. The resulting alkynyl coumarin were converted into chromans or tetrahydrofuro[2,3b]benzofurans with the characteristic framework of fungal metabolite aflatoxins (Scheme 58).

The zinc mediated conjugated alkynylation has been also applied by Blay and Pedro to achieve the first enantioselective conjugate alkynylation of $\beta$, $\beta$-disubstituted enones. Thus, $\beta$-aryl$\beta$-trifluoromethyl enones were alkynylated with a terminal alkyne and diethylzinc in the presence of $3,3^{\prime}$-bis(perfluorophenyl)BINOL as the chiral ligand to give the corresponding ketones bearing a trifluoromethylated propargylic quaternary stereocenter with fair to good enantioselectivities. (Scheme 59). ${ }^{62}$ 

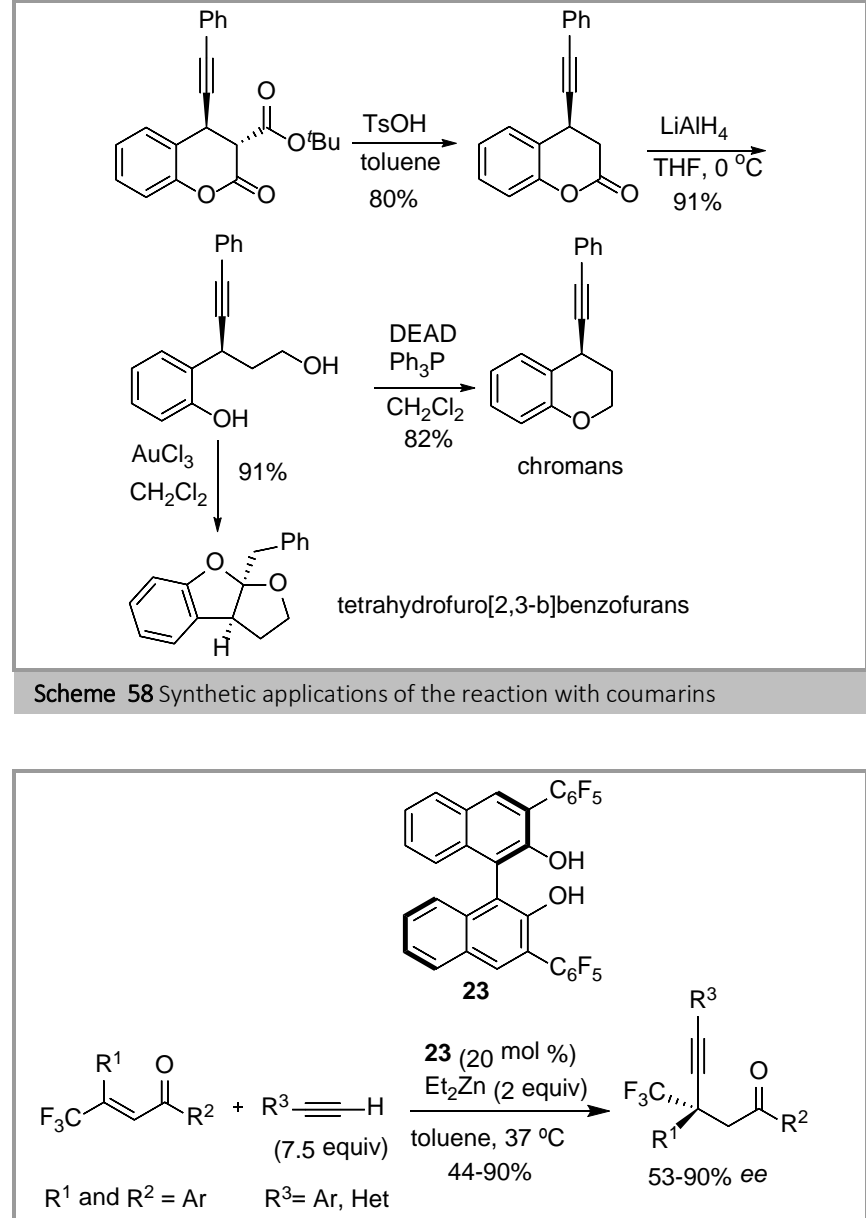

Scheme 59 First enantioselective conjugate alkynylation of $\beta, \beta$-disubstituted enones

3.2.7. Palladium and ruthenium catalyzed conjugate alkynylations

During their study on $\mathrm{Pd}(0)$-catalyzed conjugate alkynylation of enones, the group of Mascareñas reported two examples of an asymmetric version using a chiral BINOL-derived phosphite obtaining the expected alkynylation products, but with low enantioselectivity (Scheme 60). ${ }^{29}$

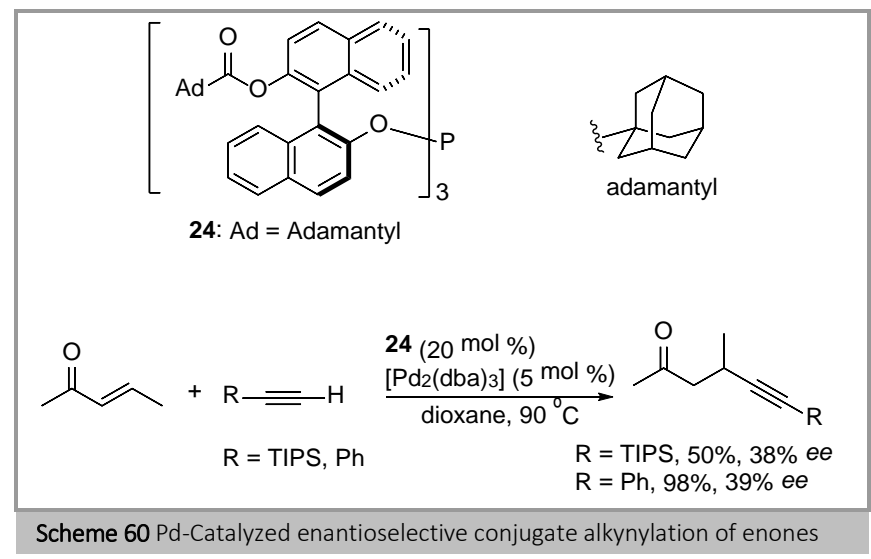

On the other hand, Ito reported the enantioselective addition of phenylacetylene to $(E)$-pent-3-en-2-one in $49 \%$ yield and $82 \%$ $e e$, employing a Ru-phebox complex as catalyst (Scheme 61). ${ }^{27}$

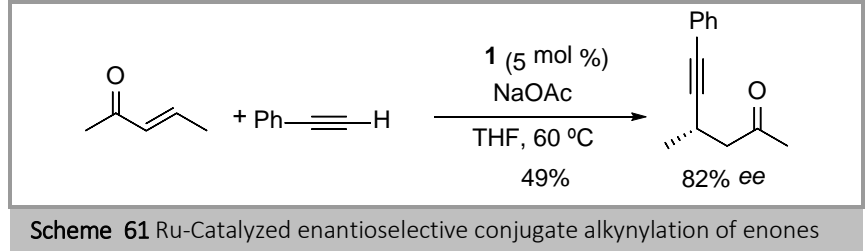

\section{Non-stereoselective and enantioselective alkynylation of other electrophilic alkenes}

\subsection{Conjugate alkynylation of nitroalkenes}

Examples of alkynylation of double bonds conjugate with electron-withdrawing groups other than carbonyl are scarce. Despite this, the first example of alkynylation of nitroalkenes was reported as early as in 1978 by Mechkov, who carried out the addition of phenylethynylmagnesium bromide to nitroethene, nitrostyrene or 1-nitropentene in $32-51 \%$ yields. ${ }^{63}$ Later in 1989, Pecunioso described the alkynylation of several cyclic and acyclic nitroalkenes, by means of ether-free diethylalkynylalanes, which had been prepared in a hexane solution by adding either alkynyllithium to $\mathrm{Et}_{2} \mathrm{AlCl}$ or the suitable alkyne to $\mathrm{Et}_{3} \mathrm{Al}$ (Scheme 62). ${ }^{64}$

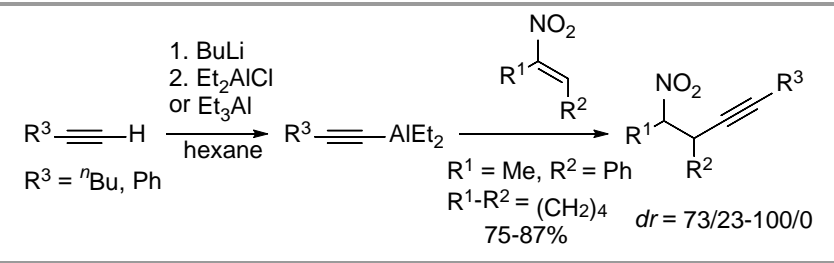

Scheme 62 Non-stereoselective conjugate alkynylation of nitroalkenes with alkynyldiethylaluminum reagents

Recently, as a part of a larger study on 1,4-conjugate addition of Grignard reagents to nitrodienes, Dieter described the reaction of alkynyl Grignard reagents with 1-nitro-1,3-pentadiene catalyzed by $\mathrm{Zn}(\mathrm{II})$ salts. In the presence of $\mathrm{ZnBr}_{2}$ 1hexynylmagnesium bromide gave exclusively the 1,4-adduct in $\mathrm{Et}_{2} \mathrm{O}$, while the more electron deficient phenylethynylmagnesium bromide gave good yields but poor regioselectivity under similar conditions. However, the use of $\mathrm{Zn}(\mathrm{CN})_{2}$ instead of $\mathrm{ZnBr}_{2}$ led to excellent yields and regioselectivity for the major 1,4-adduct, with this alkyne (Scheme 63). ${ }^{65}$

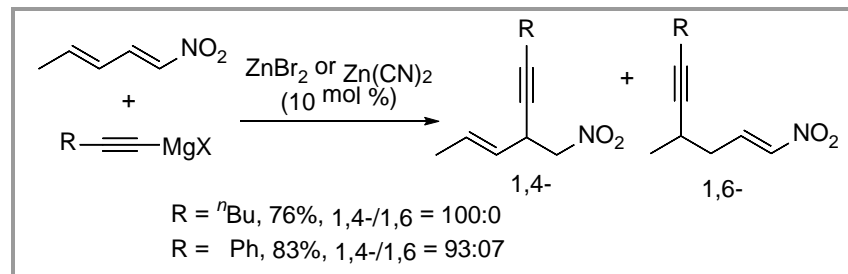

Scheme 63 1,4-Regioselective alkynylation of nitrodienes with alkynyl Grignard reagents catalyzed by zinc

In 2005, Tomioka described the first enantioselective alkynylation of nitroalkenes. The reaction was carried out by 
using terminal alkynes and dialkylzinc in the presence of 3 equivalents of a $\quad(1 R, 2 R)$-2-(dimethylamino)-1,2diphenylethanol as chiral promoter. Addition of galvinoxyl was required to avoid formation of radical species that were detrimental to yield. The addition of arylacetylenes to nitrocyclohexene was carried out. Diastereoselectivity depended on the quenching procedure to favor the trans (ammonium chloride) or cis $(\mathrm{AcOH})$ diastereomer. The reaction products were obtained with good yields and high enantiomeric excesses, especially for the trans isomer. Linear nitroalkenes having alkyl groups at the $\beta$-position were suitable substrates, although moderate yields were obtained with substrates having a $\gamma-\mathrm{H}$, due to concomitant deprotonation and isomerization of

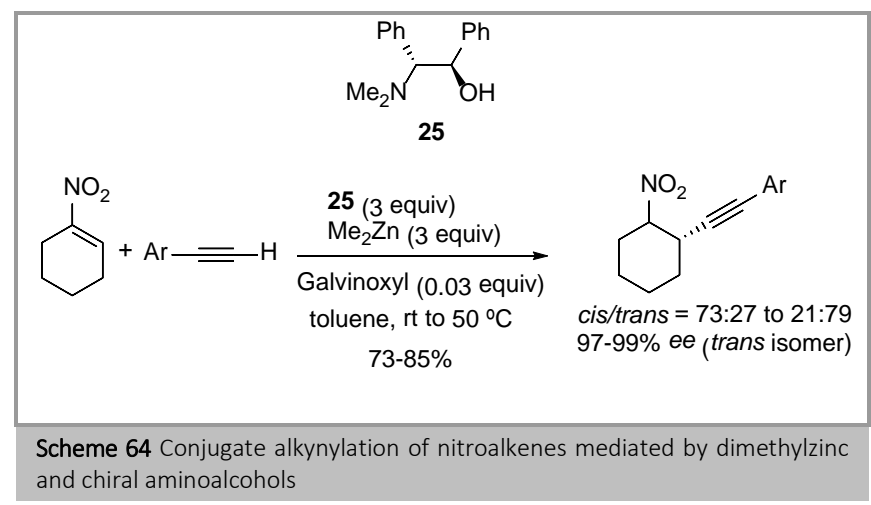

Finally, the $\mathrm{Rh}(\mathrm{I})$-catalyzed enantioselective alkynylation of nitroalkenes has been reported by Nishimura and Hayashi authors for the alkynylation of enals.52 Alkyl and substituted aryl groups were tolerated as substituents at the $\beta$-position of the nitroalkene, providing the corresponding $\beta$-alkynyl nitrocompounds with high yield and enantioselectivity (Scheme 65). ${ }^{67}$

Scheme 65 Rh(I)-catalyzed enantioselective alkynylation of nitroalkenes

\subsection{Conjugate alkynylation of unsaturated sulfones and sulfoxides}

Alkynylation of double bonds conjugated with sulfur electronwithdrawing functionalities are very scarce. Isobe developed a methodology for the asymmetric addition of alkynyllithium reagents to silylvinyl sulfones assisted by a chiral template attached to the double bond (Scheme 66). ${ }^{68}$ Examples by these authors included the use of valinol-oxazolidine, cyclic alcohols or sugars as templates. In this late case, by protecting one of the $\mathrm{OH}$ groups in the template a diastereodivergent alkynylation was made possible. ${ }^{68 c, d}$ This methodology has been extended by the authors to other silylvinyl sulfones and more complex double bond (Scheme 64). ${ }^{66}$ under the same conditions developed previously by these

functionalized alkynes and applied to the synthesis of different fragments of the marine toxin ciguatoxin. 69

The same template-assisted conjugate addition methodology has been applied by the group of Isobe in the synthesis of chiral cyclobutanes, starting with a diastereoselective conjugate alkynylation, followed by a Lewis acid assisted intramolecular epoxide cleavage (Scheme 67). ${ }^{70}$ The use of $\mathrm{LiBr} / \mathrm{NaBr}$ as additive during the alkynylation step was essential to obtain full diastereoselectivity.
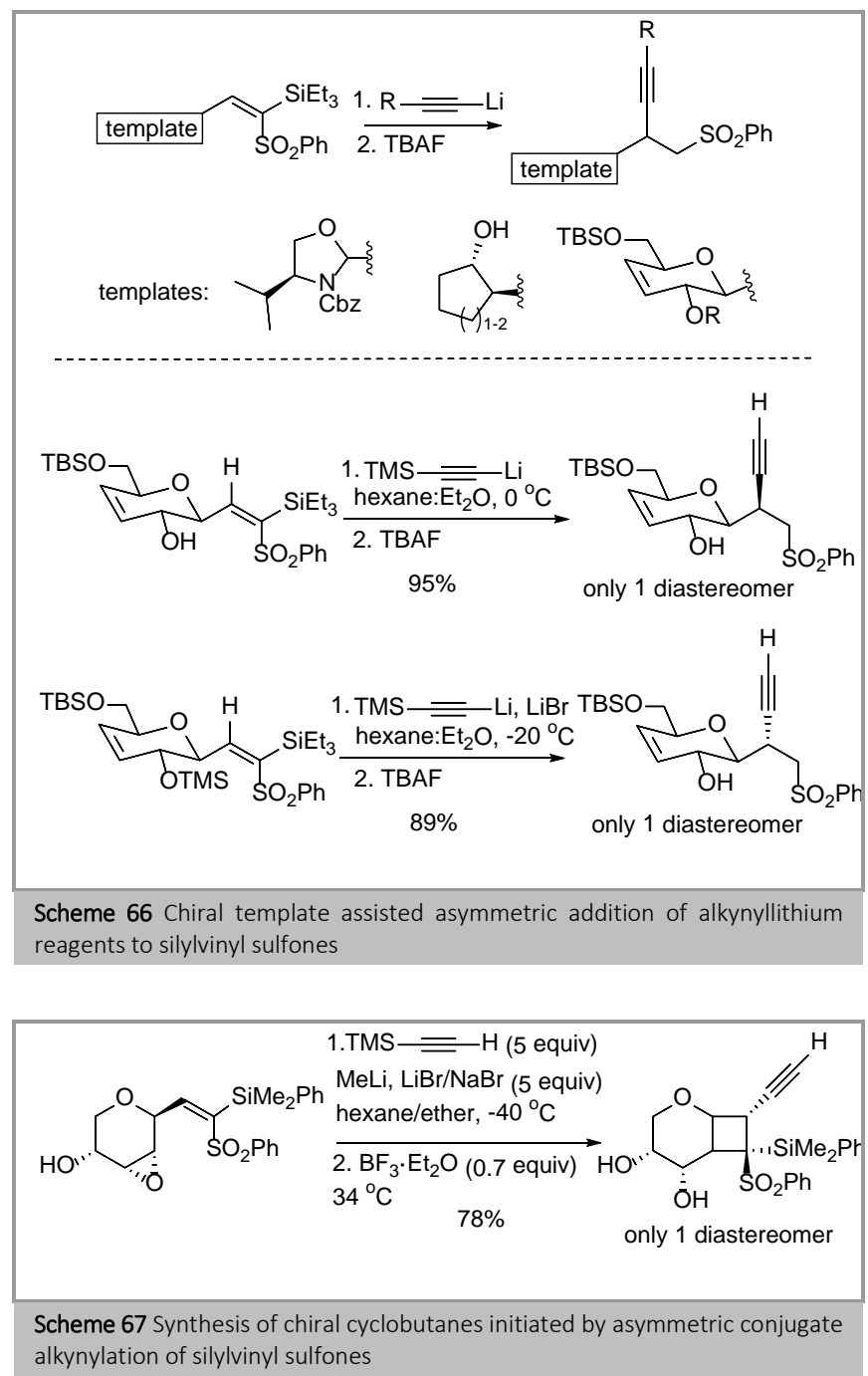

Recently, the group of Yanai has described the regioselective alkynylation of 1,1-bis(triflyl)alkadienes. In the only described example ethynylmagnesium bromide reacted with 1,1bis(triflyl)-4-phenyl1,3-butadiene in the presence of 1.2 equivalents of $\mathrm{CeCl}_{3}$ to give the $\beta$-alkynylated product with $67 \%$ yield and 8.2:1 regioisomeric ratio. In absence of the cerium salt, low regioselectivity was observed (Scheme 68). ${ }^{71}$

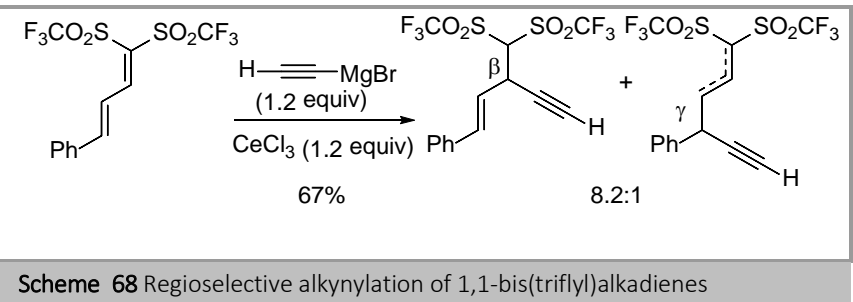

Template for SYNTHESIS @ Thieme Stuttgart · New York 2020-09-29 
Finally, Sato has reported the alkynylation of 1-chlorovinyl $p$ tolyl sulfoxides with lithium alkynylides to give the corresponding adducts in moderate to good yields. Further treatment of these adducts with Grignard reagents resulted in formation of conjugated enynes via the 1,2-carbon-carbon insertion reaction of the generated magnesium carbene intermediates (Scheme 69). ${ }^{72}$

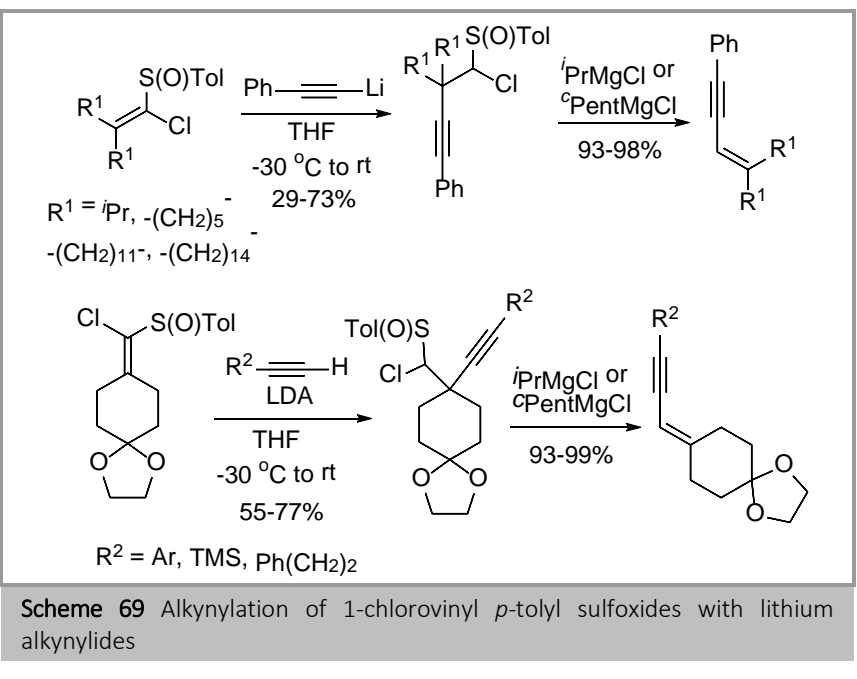

\subsection{Conjugate alkynylation of unsaturated phosphonates and phosphine oxides}

The addition of sodium acetylide to diethyl ethylidene bisphosphonate or ethyl 1-ethoxycarbonyl ethylidene phosphonate has been used by several authors to obtain good yields of propargylic phosphonates, which are useful to prepare new drug candidates and bioconjugates through "click chemistry" (Scheme 70). ${ }^{73}$

\begin{tabular}{|l}
$\mid$ \\
Scheme 70 Addition of sodium acetylide to conjugate phosphonates \\
$\begin{array}{l}\mathrm{X}=\mathrm{THF},-20{ }^{\circ} \mathrm{C} \\
\mathrm{X}=\mathrm{CO}_{2} \mathrm{Et}, 74 \%\end{array}$
\end{tabular}

As a part of their study on conjugate alkynylation of carbonyl compounds catalyzed by the PHEBOX Ru acetate complex, ${ }^{27}$ Ito and Nishiyama reported that the same catalyst was able to promote the addition of a terminal alkyne (phenylacetylene) to diethyl vinylphosphonate affording the corresponding $\beta$-alkynyl phosphonate in 67\% yield (Scheme 71).

\begin{tabular}{|l}
\hline Scheme 71 Ru-catalyzed addition of phenylacetylene to diethyl \\
vinylphosphonate
\end{tabular}

On the other hand, Nishimura and Hayashi developed an asymmetric addition of terminal alkynes to diarylphosphinylallenes giving exo-enynes in high yields with high regio- and enantioselectivity, using a binap-Rh(I) complex as catalyst and diphenylphosphinic acid as a proton source. The reaction of 1-substituted phosphinylallenes with (triphenylsilyl)acetylene or terminal alkynes featuring a propargylic ether gave the corresponding enynes in good yields with high regio- and enantioselectivity. The asymmetric addition also proceeded with simple terminal alkynes, 1-octyne and phenylacetylene, although the yields of the enynes were somewhat lower (Scheme 72). ${ }^{74}$

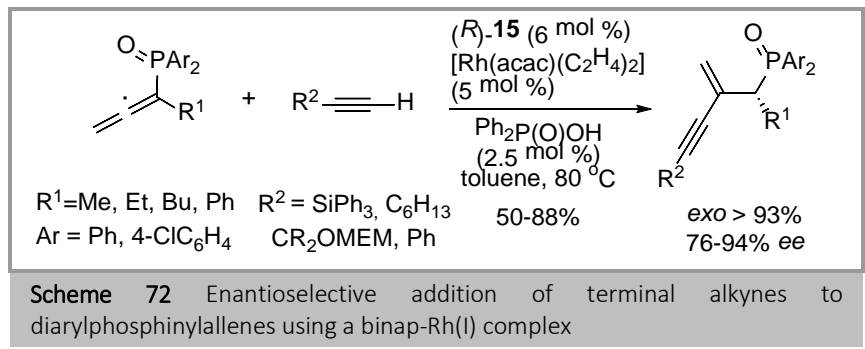

Based on mechanistic studies, the catalytic cycle outlined in Scheme 73 was proposed. The cycle involves an alkynylrhodium(I) complex that after insertion of the allene into the rhodium-carbon bond forms a $\pi$-allylrhodium(I)complex. Protonolysis of this complex with an acid (HY) would then furnish the enyne product and a rhodium(I) species bearing the anionic ligand resulting from the acid. The reaction of this species with the alkyne would regenerate the alkynylrhodium and the acid (HY), giving continuity to catalysis.

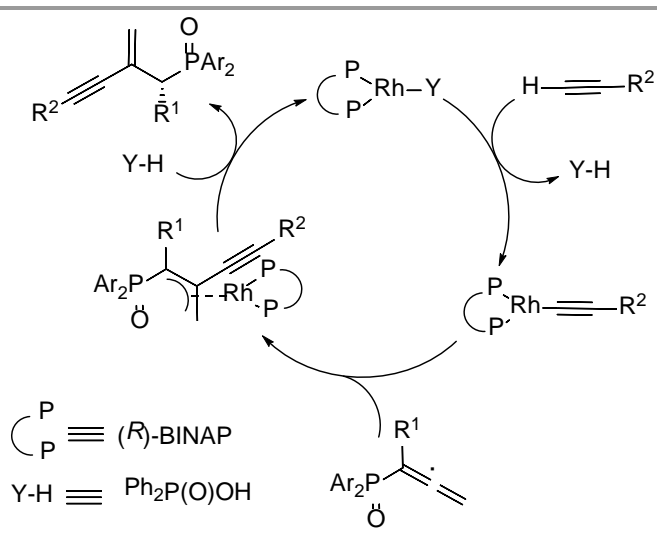

Scheme 73 Proposed catalytic cycle for the addition of terminal alkynes to diarylphosphinylallenes

\section{5. $\gamma$-Alkynylation of $\alpha, \beta$-unsaturated amides and $\delta$-alkynylation of electrophilic dienes}

While attempting to perform the conjugate alkynylation of unsaturated amides with triisopropylacetylene under iridium catalysis, the group of $\mathrm{Li}$ found that, unexpectedly, the alkynylation occurred regio- and enantioselectively at $\gamma$ instead of $\beta$-position. The reaction was catalyzed by a complex formed from $\operatorname{Ir}(\mathrm{COD})_{2} \mathrm{OTf}(\mathrm{COD}=$ cyclooctadienyl) and $(R)$-DMSegphos, and allowed great variation at the amide nitrogen atom, being of application to secondary and tertiary alkenyl amides. A variety of functionalized alkyl substituents at the $\beta$-position were also 
tolerated; however no reaction was observed with cinnamides (Scheme 74). ${ }^{75}$

To account for the unexpected $\gamma$-selectivity the authors proposed a mechanism involving oxidative addition of the terminal alkyne to the bisphosphine-ligated Ir center to give an alkynyl iridium hydride that, after conjugate addition to the amide would form a five-membered iridacycle in which the $\beta$ carbon of the amide is attached to Ir. This intermediate would undergo a $\beta$-hydride elimination to give another iridium complex coordinated to a $\beta, \gamma$-unsaturated amide that after irreversible migratory insertion of the alkene and reductive elimination would provide the $\gamma$-alkynylation product (Scheme 75).
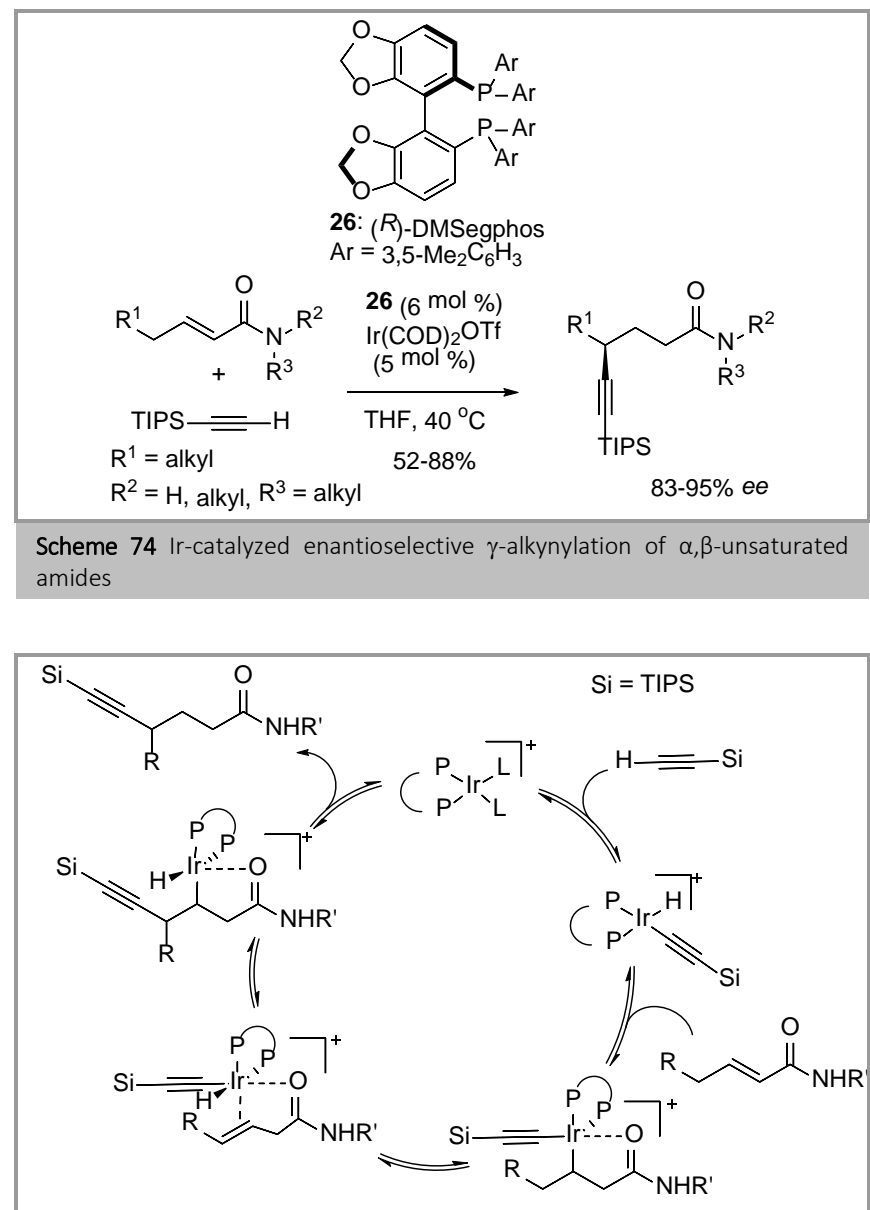

Scheme 75 Proposed catalytic cycle for the Ir-catalyzed $\gamma$-alkynylation of $\alpha, \beta$ unsaturated amides

On the other hand, the addition of terminal alkynes to extended conjugate systems such as $\alpha, \beta, \gamma, \delta$-unsaturated carbonyl compounds is challenging due to the difficulty of controlling the regioselectivity as well as the enantioselectivity. Watanabe reported the ruthenium-catalyzed addition of terminal alkynes to 1,3-dienes, which included a few examples of formal 1,6addition to dienoates. ${ }^{76}$ As a part of synthetic studies toward 7substituted estradiol derivatives carried out in 2007, van Lier performed the 1,6-conjugate alkynylation of 19-nordehydrotestosterone using ethynyl magnesium bromide in the presence of $\mathrm{CuCl}$ catalyst, obtaining the 1,6-alkynylation product in $60 \%$ yield with complete regio- and stereoselectivity (Scheme 76). ${ }^{77}$
Nishimura and Hayashi have described the only example of regio- and enantioselective 1,6-addition of terminal alkynes to linear $\alpha, \beta, \gamma, \delta$-unsaturated carbonyl compounds. The addition of (triisopropylsilyl)acetylene to dienoates and dienamides having alkyl substituents at the $\delta$ position was catalyzed by a $\mathrm{Co}(\mathrm{I}) /$ duphos complex in the presence of $\mathrm{Zn}$ to reduce Co(II) to Co(I). The reaction took place with exclusive 1,6-regioselectivity and the corresponding $\delta$-alkynylated $\alpha, \beta$-unsaturated esters and amides were obtained with high enantioselectivity (Scheme 77)..$^{78}$

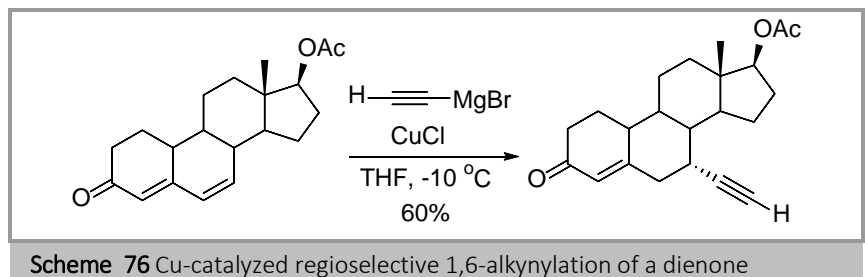

Scheme $76 \mathrm{Cu}$-catalyzed regioselective 1,6-alkynylation of a dienone

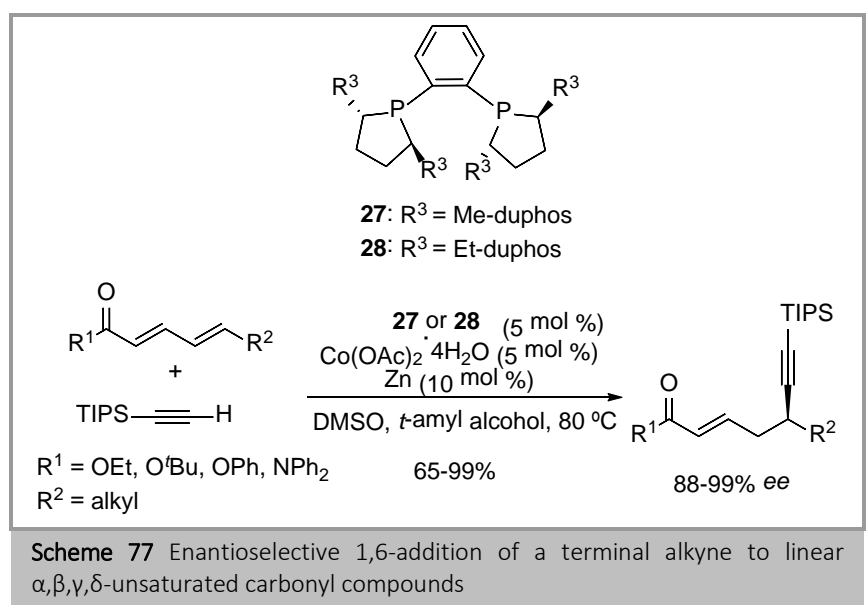

The geometrical structure of the starting dienoate affected both the absolute configuration of the product and the reactivity of the dienoate. Thus, the $(2 E, 4 E)$ isomer gave the product with the opposite configuration to that obtained from the $(2 E, 4 Z)$ isomer. On the other hand, the $(2 Z, 4 Z)$ isomer gave the same product as the $(2 E, 4 Z)$ isomer but with low yield and enantioselectivity, while the $(2 Z, 4 E)$ isomer gave mixtures of two cis/trans isomers.

On the basis of experimental research, the authors proposed the catalytic cycle illustrated in Scheme 78. The catalytic reaction would be initiated by the reduction of cobalt(II) to cobalt(I) by zinc giving cobalt(I) acetate, which would undergoes the reaction with a terminal alkyne to an alkynylcobalt(I) intermediate. Coordination of this to the dienoate with a cisoid diene moiety would result in the formation of ( $\eta 4$-diene)-cobalt complex, which after insertion of the diene into the alkynyl cobalt bond would give a $\pi$-allylcobalt that, after protonation at the $\gamma$-position by an alkyne would lead to the reaction product an re-start the catalytic cycle.

\section{Alternative enantioselective procedures}

A number of methods to obtain $\beta$-alkynyl carbonyl compounds in an enantioselective manner, which do not involve the 
conjugate addition of alkynyl species to conjugated double bonds have been reported in the literature.

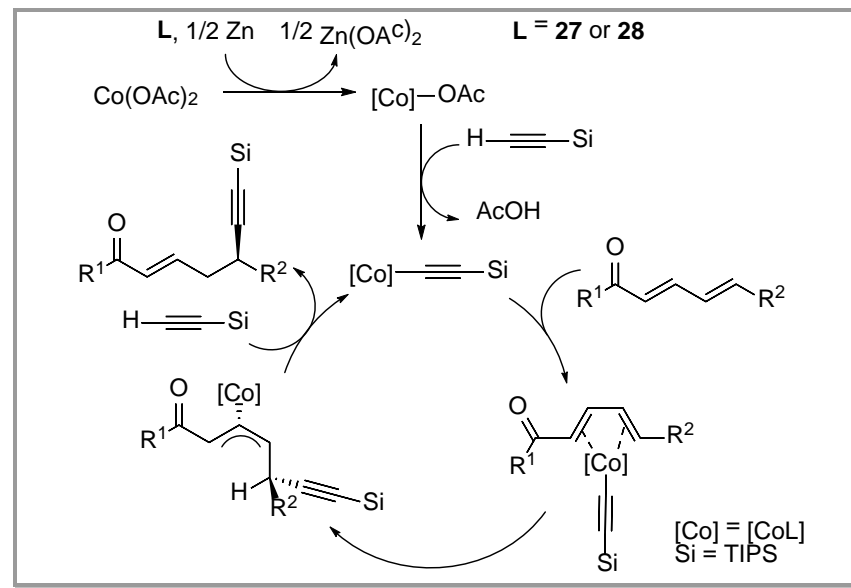

Scheme 78 Proposed mechanism for the Co(I)-catalyzed 1,6-conjugate alkynylation of dienoates

Trost has developed a sequential catalysis strategy for the synthesis of chiral alkynyl carbonyl and sulfonyl derivatives. The sequence involves coupling of terminal alkyne donors with acetylenic esters, ketone or sulfone acceptors catalyzed by Pd/TDMPP (TDMPP = tris(2,6-dimethoxyphenylphosphine)), followed by a regio- and enantioselective conjugate reduction of the double bond with a silane in the presence of a copper/walphos complex (Scheme 79). ${ }^{79}$

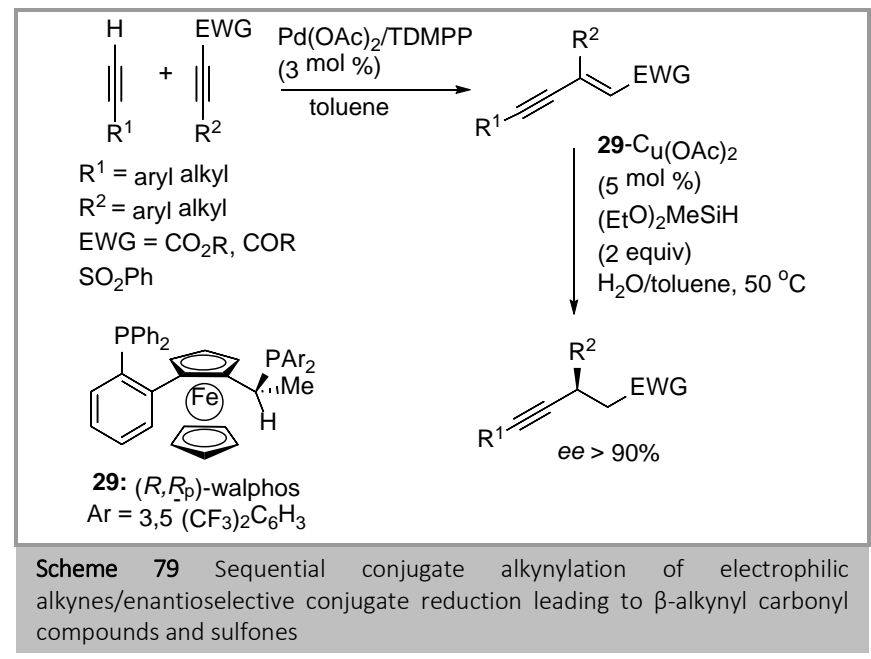

Two organocatalytic approaches to chiral $\beta$-alkynyl carbonyl compounds have been described. Jørgensen has made use of $\beta$ keto heterocyclic sulfones as synthetic equivalents of alkynes. In a first work, conjugate addition of the ketosulfone to unsaturated aldehydes was carried out via iminium activation with the Jørgensen-Hayashi catalyst to give the corresponding adducts with high enantioselectivity. Then, the ketosulfone moiety was converted into an alkyne through a Smiles rearrangement in mild basic medium. Protection of the aldehyde as diethylacetal needed to be conducted before treatment with base to avoid side reactions. The authors also developed a protocol for cyclic enones using in this case the quinine-2TfOH salt as catalyst in the conjugate addition step. (Scheme 80). ${ }^{80}$
On the other hand, Jurberg has employed alkyliden isoxazol-5ones which can undergo aminocatalyzed conjugate addition of ketones in the presence of a quinine-derived amine. The resulting oxazolinones can be converted into alkynes via a nitrosative degradation (Zard reaction) to give chiral $\beta$-alkynyl ketones (Scheme 81). ${ }^{81}$ The target compounds can be accessed in broad scope, in moderate to good yields, perfect diastereocontrol and good to excellent enantioselectivity.

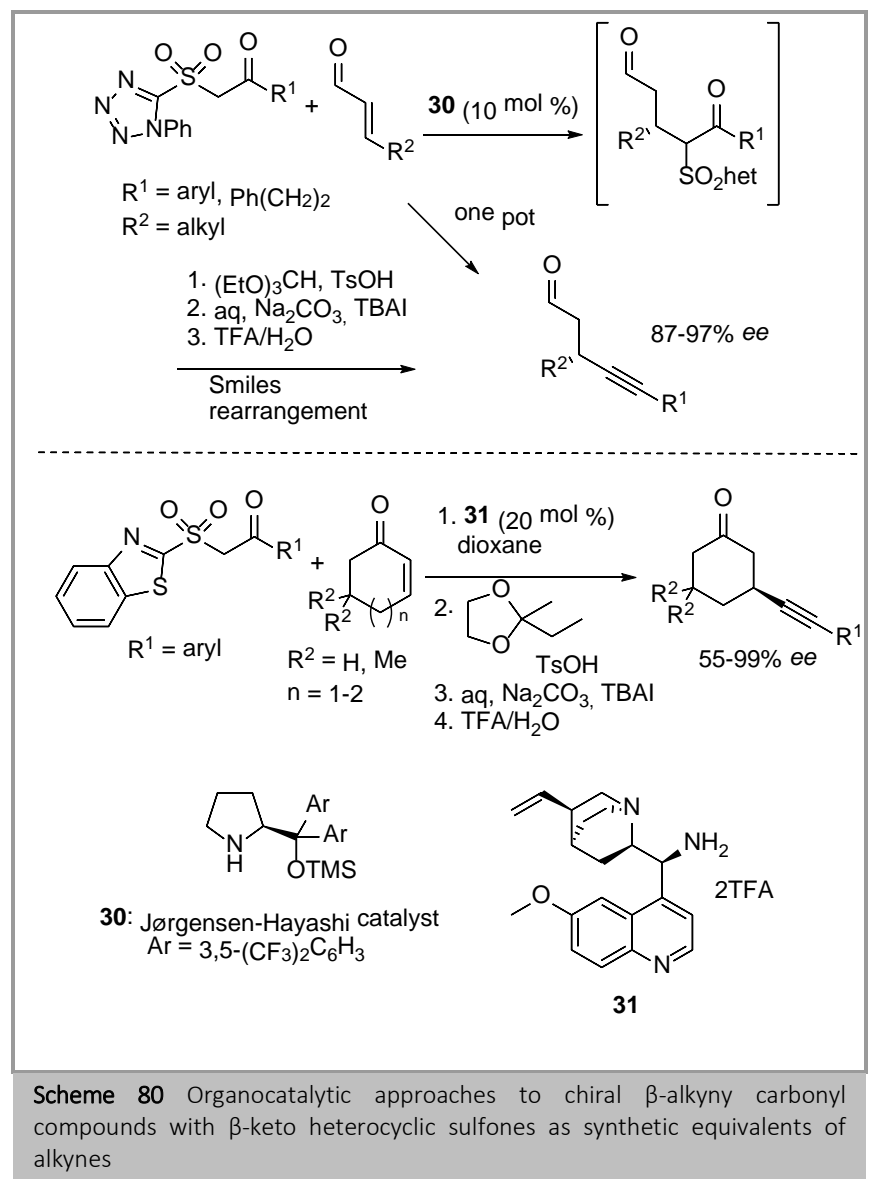

Scheme 81 Organocatalytic approach to chiral $\beta$-alkyny carbonyl compounds
from alkyliden isoxazol-5-ones




\section{Conclusion and outlook}

The initial development of the conjugate alkynylation of carbonyl compounds was hampered by the preference of organolithium and Grignard reagents to experiment 1,2addition to the carbonyl group and by the reluctance of organocopper reagents, the preferred organometallics for conjugate addition, to transfer the alkyne ligand. Early success was obtained with uncatalyzed additions of other metalated alkynes. The use of additives and metal catalysts facilitated the reaction of pre-formed metal alkynylides first, and of terminal alkynes later. These methods paved the way to the development of enantioselective procedures that have experienced an enormous growth in the last decade. Catalysis by copper, zinc, rhodium or cobalt, has permitted highly enantioselective reactions using terminal alkynes. Despite these progresses, serious limitations still remain: Substrate scope is narrow, in most of the cases doubly activated alkenes or special substrates are required, alkyne scope is also limited, many of the reported reactions only work with silylalkynes or with phenyl acetylene derivatives, while the results are usually low with aliphatic alkynes; furthermore many of the procedures still require chiral ligands which are difficult to prepare or require expensive precious metals. It is expected that future research will address these drawbacks as well as the extension, particularly of enantioselective methods, to other less studied electrophilic double bonds such as nitroalkenes, unsaturated phosphonates and sulfones, and so on.

\section{Funding Information}

Ministerio de Economía, Industria y Competitividad and FEDER. Grant CTQ2017-84900-P.

Generalitat Valenciana and Fondo Social Europeo. Grant APOST-2016139.

\section{Acknowledgment}

A. S.-M thanks the Generalitat Valenciana and Fondo Social Europeo for a post-doctoral grant. Access to the facilities of "Servei d'Informació Bibliogràfica de la Universitat de València" is acknowledged.

\section{References}

(1) (a) Bohlmann, F.; Burkhardt, H.; Zdero, C. Naturally Occurring Acetylenes, Academic Press: New York, 1973. (b) Jones, E. R. H.; Thaller, V. In The Chemistry of the Carbon-Carbon Triple Bond, Part 2; Patai, S., Ed.; Wiley: New York, 1978, 621.

(2) (a) Review: Nielsen, M. B.; Diederich, F. Chem. Rev. 2005, 105, 1837. (b) Wan, W. B.; Brand, S. C.; Pak, J. J.; Haley, M. M. Chem. Eur. J. 2000, 6, 2044. (c) West, K.; Wang, C.; Batsanov, A. S.; Bryce, M. R. Org. Biomol. Chem. 2008, 6, 1934.

(3) (a) Hudrlik, P. F.; Hudrlik, A. M. In Applications of Acetylenes in Organic Synthesis, in The Chemistry of the Carbon-Carbon Triple Bond Part 1; Patai, S., Ed.; Wiley: Chichester, 1978, 199. (b) Modern Alkyne Chemistry. Catalytic and Atom Economy Reactions; Trost, B. M., Ed.; Wiley-VCH: Weinheim, 2015. (c) Fanga, G.; Bi, X. Chem. Soc. Rev. 2015, 44, 8124. (d) Salvio, R.; Moliterno, M.; Bella, M. Asian J. Org. Chem. 2014, 3, 340. (e) Trotus,I.-T.; Zimmermann, T.; Schüth, F. Chem. Rev. 2014, 114, 176. (f) Chinchilla, R.; Nájera, C. Chem. Rev. 2014, 114, 1783.

(4) (a) Trost, B. M.; Weiss, A. Adv. Synth. Catal. 2009, 351, 963. (b) Li, C.-J. Acc. Chem. Res. 2010, 43, 581.

(5) Blay, G.; Monleón, A.; Pedro, J. R. Curr. Org. Chem. 2009, 13, 1498.

(6) Fujimori, S.; Knöpfel, T. F.; Zarotti, P.; Ichikawa, T.; Boyall, D.; Carreira, E. M. Bull. Chem. Soc. Jpn. 2007, 80, 1635.
(7) Despite this, the 1,4-addition of simple organolithium to enones highly hindered in the proximity of the carbonyl group such as 1,1,1-triphenyl-2-penten-1-one, as well as the conjugate alkynylation of 2-aryliden-1,3-diesters with alkynyl Grignard reagents have been reported. See (a) Locher, R.; Seebach, D. Angew. Chem. 1981, 93, 614 and (b) Kruse, L. I.; Kaisr, C.; De Wolf, W. E.; Chambers, P. A.; Goodhart, P. J.; Ezekiel, M.; Ohlstein, E. H. J. Med. Chem. 1988, 31, 704, respectively.

(8) Hooz, J.; Layton, R. B. J. Am. Chem. Soc. 1971, 93, 7320.

(9) (a) Pappo, R.; Collins, P. W. Tetrahedron Lett. 1972, 2627. (b) Bruhn, M.; Brown, C. H.; Collins, P. W.; Palmer, J. R.; Dayanl, E. Z.; Pappo, R. Tetrahedron Lett. 1976, 235.

(10) (a) Hansen, R. T.; Carr, D. B.; Schwartz, J. J. Am. Chem. Soc. 1978, 100, 2244. (b) Schwartz, J.; Carr, D. B.; Hansen, R. T.; Dayrit, F. M. J. Org. Chem. 1980, 45, 3053.

(11) Maruoka, K.; Shimada, I.; Imoto, H.; Yamamoto, H. Synlett 1994, 519.

(12) Ahmar, S.; Fillion, E. Org. Lett. 2014, 16, 5748.

(13) Sinclair, J. A.; Molander, G. A.; Brown, H. C. J. Am. Chem. Soc. 1977, 99, 954.

(14) Bertoline, F.; Woodward, S. Synlett 2009, 51.

(15) Kim, S.; Lee, J. M. Tetrahedron Lett. 1990, 7627.

(16) Kim, S.; Park, J. H.; Jon, S. Y. Bull. Korean Chem. Soc. 1995, 16, 783.

(17) Eriksson, M.; Iliefski, T.; Nilsson, M.; Olsson, T. J. Org. Chem. 1997, $62,182$.

(18) Baars, H.; Classen, M. J.; Aggarwal, V. K. Org. Lett. 2017, 19, 6008.

(19) Daia, D. E.; Gabbutt, C. D.; Heron, B. M.; Hepworth, J. D.; Hursthouse, M. B.; Malik, K. M. A. Tetrahedron Lett. 2003, 44, 1461.

(20) Xu, Y.; Pan, Y.; Liu, P.; Wang, H.; Tian, X.; Su, G. J. Org. Chem. 2012, $77,3557$.

(21) Shibata, I.; Kano, T.; Kanazawa, N.; Fukuoka, S.; Baba, A. Angew. Chem. Int. Ed. 2002, 41, 1389.

(22) Kovalev, I. P.; Nikishin, G. I. Tetrahedron Lett. 1990, 31, 7063.

(23) Lerum, R. V.; Chisholm, J. D. Tetrahedron Lett. 2004, 45, 6591.

(24) Picquet, M.; Bruneau, C.; Dixneuf, P.H. Tetrahedron 1999, 55, 3937.

(25) Chang, S.; Na, Y.; Choi, E.; Kim, S. Org. Lett. 2001, 3, 2089.

(26) (a) Nishimura, T.; Washitake, Y.; Nishiguchi, Y.; Maeda, Y.; Uemura, S. Chem. Commun. 2004, 1312. (b) Nishimura, T.; Washitake, Y.; Uemura, S. Adv. Synth. Catal. 2007, 349, 2563.

(27) Ito, J.; Fujii, K.; Nishiyama, H. Chem. Eur. J. 2013, 19, 601.

(28) (a) Chen, L.; Li, C-J. Chem. Commun. 2004, 2362. (b) Zhou, L.; Chen, L.; Skouta, R.; Jiang, H.-F.; Li, C.-J. Org. Biomol. Chem. 2008, 6, 2969.

(29) Villarino, L.; García-Fandiño, R.; López, F., Mascareñas, J. L. Org. Lett. 2012, 14, 2996.

(30) (a) Knöpfel, T. F.; Carreira, E. M. J. Am. Chem. Soc. 2003, 125, 6054. (b) Fujimori, S.; Carreira, E. M. Angew. Chem. Int. Ed. 2007, 46, 4964.

(31) Li, S.; Jia, W.; Jiao, N. Adv. Synth. Catal. 2009, 351, 569.

(32) Rajesh, N.; Prajapati, D. Org. Biomol. Chem. 2015, 13, 4668.

(33) Kidway, M.; Jain, A.; Bhardwaj, S. Catal. Lett. 2011, 141, 183.

(34) (a) Carreño, M. C.; Pérez González, M.; Ribagorda, M.; Fischer, J. J. Org. Chem. 1996, 61, 6758. (b) Carreño, M. C.; Pérez González, M.; Ribagorda, M.; Houk, K. N. J. Org. Chem. 1998, 63, 3687.

(35) Chong, J. M.; Shen, L.; Taylor, N. J. J. Am. Chem. Soc. 2000, 122, 1822.

(36) Knöpfel, T. F.; Boyall, D.; Carreira, E. M. Org. Lett. 2004, 13, 2281.

(37) (a) Cui, S.; Walker, S. D.; Woo, J. C. S.; Borths, C. J.; Mukherjee, H.; Chen, M. J.; Faul, M. M. J. Am. Chem. Soc. 2010, 132, 436. (b) Woo, J. C. S.; Cui, S.; Walker, S. D.; Faul, M. M. Tetrahedron 2010, 66, 4730.

(38) Kwak, Y.-S.; Corey, E. J. Org. Lett. 2004, 6, 3385.

(39) Larionov, O. V.; Corey, E. J. Org. Lett. 2010, 12, 300.

(40) Wu, T. R.; Chong, J. M.; J. Am. Chem. Soc. 2005, 127, 3244.

(41) Pellegrinet, S. C.; Goodman, J. M. J. Am. Chem. Soc. 2006, 128, 3116.

(42) Knopfel, T. F.; Zarotti, P.; Ichikawa, T.; Carreira, E. M. J. Am. Chem. Soc. 2005, 127, 9682.

(43) Zarotti, P.; Knöpfel, T. F.; Aschwanden, P.; Carreira, E. M. ACS Catal. 2012, 2, 1232

(44) Nishra, S.; Liu, J.; Aponick, A. J. Am. Chem. Soc. 2017, 139, 3352. 
(45) (a) Yazaki, R.; Kumagai, N.; Shibasaki, M. J. Am. Chem. Soc. 2010 132, 10275. (b) Yazaki, R.; Kumagai, N.; Shibasaki, M. Chem. Asian.J. 2011, 6, 1778

(46) Yazaki, R.; Kumagai, N.; Shibasaki, M. Org. Lett. 2011, 13, 952.

(47) Sanz-Marco, A.; García-Ortiz, A.; Blay, G.; Fernández, I.; Pedro, J. R. Chem. Eur. J. 2014, 20, 668

(48) (a) Sanz-Marco, A.; Blay, G.; Muñoz, M. C.; Pedro, J. R. Chem. Commun. 2015, 51, 8958. (b) Sanz-Marco, A.; Blay, G.; Muñoz, M. C.; Pedro, J. R. Chem. Eur. J. 2016, 22, 10057.

(49) Sanz-Marco, A.; García-Ortiz, A.; Blay, G.; Pedro, J. R. Chem. Commun 2014, 50, 2275 .

(50) Nishimura, T.; Kato, T.; Takatsu, K.; Shintani, R.; Hayashi, T. J. Am Chem. Soc. 2007, 129, 14158

(51) Nishimura, T.; Guo, X-X.; Uchiyama, N.; Katoh, T.; Hayashi, T. J. Am. Chem. Soc. 2008, 130, 1576.

(52) Nishimura, T.; Sawano, T.; Hayashi, T. Angew. Chem. Int. Ed. 2009 48, 8057.

(53) Mandelalide A: (a) Brutsch, T. M.; Bucher, P.; Altmann, K.-H. Chem Eur. J. 2016, 22, 1292. Aphanamol I: (b) Ferrara, S. J.; Burton, J. W. Chem. Eur. J. 2016, 22, 11597.

(54) Nishimura, T.; Tokuji, S.; Sawano, T.; Hayashi, T. Org. Lett. 2009, 11 3222.

(55) Dou, X.; Huang, Y.; Hayashi, T. Angew. Chem. Int. Ed. 2016, 55, 1133.

(56) Zhi, Y.; Huang, J.; Liu, N.; Lu, T.; Dou, X. Org. Lett. 2017, 19, 2378.

(57) Choo, K.-L.; Lautens, M. Org. Lett. 2018, 20, 1380.

(58) Fillion, E.; Zorzitto, A. K. J. Am. Chem. Soc. 2009, 131, 14608.

(59) Nishimura, T.; Sawano, T.; Ou, K.; Hayashi, T. Chem. Commun. 2011, 47, 10142.

(60) Blay, G.; Cardona, L.; Pedro, J. R.; Sanz-Marco, A. Chem. Eur. J. 2012 $18,12966$.

(61) Blay, G.; Muñoz, M. C.; Pedro, J. R.; Sanz-Marco, A. Adv. Synth. Catal. 2013, 355, 1071.

(62) Sanz-Marco, A.; Blay, G.; Vila, C.; Pedro, J. R. Org. Lett. 2016, 18 3538.

(63) Mechkov, Ts. D.; Sulimov, I. G.; Usik, N. V.; Perekalin, V. V.; Mladenov, I. Zhu. Org. Khim. 1978, 14, 733.

(64) Pecunioso, A.; Menicagli, R. J. Org. Chem. 1989, 54, 2391.

(65) Dhakal, R. C.; Dieter, R. K. Org. Lett. 2014, 16, 1362.

(66) Yamashita, M.; Yamada, K.-I.; Tomioka, K. Org. Lett. 2005, 12, 2369.

(67) Nishimura, T.; Tokuji, S.; Sawano, T.; Hayashi, T. Chem. Commun. 2010, 46, 6837

(68) (a) Isobe, M.; Hirose, Y.; Shimokawa, K.; Nishikawa, T.; Goto, T. Tetrahedron Lett. 1990, 31, 5499. (b) Herunsalee, A.; Isobe, M.; Goto, T. Tetrahedron 1991, 47, 3727. (c) Isobe, M.; Jiang, Y. Tetrahedron Lett. 1995, 36, 567. (d) Jiang, Y.; Isobe, M. Tetrahedron 1996, 52, 2877
(69) (a) Liu, T.-Z.; Kirschbaum, B.; Isobe, M. Synlett 2000, 587. (b) Baba T.; Isobe, M. Synlett 2003, 547. (c) Liu, T.-Z.; Isobe, M. Tetrahedron 2000, 56, 5391. (d) Baba, T.; Huang, G.; Isobe, M. Tetrahedron 2003, 59, 6851 .

(70) Tsao, K.-W,; Isobe, M. Org. Lett. 2010, 12, 5338

(71) Yanai, H.; Egawa, S.; Yamada, K.; Ono, J.; Aoki, M.; Matsumoto, T. Taguchi, T. Asian J. Org. Chem. 2014, 3, 556

(72) (a) Saitoh, H.; Ishida, N.; Satoh, T. Tetrahedron Lett. 2010, 51, 633 (b) Ishida, N.; Saitoh, H.; Sugiyama, S.; Satoh, T. Tetrahedron 2011 67, 3081.

(73) Skarpos, H.; Osipov, S. N.; Vorob'eva, D. V.; Odinets, I. L.; Lork, E. Roschenthaler, G. V. Org. Biomol. Chem. 2007, 5, 2361. (b) Artyushin, O.; Osipov, S. N.; Röschenthaler, G.-V.; Odinets, I. L. Synthesis 2009, 3579. (c) Massarenti, C.; Bortolini, O.; Fantin, G.; Cristofaro, D.; Ragno, D.; Perrone, D.; Marchesi, E.; Toniolo, G.; Massi, A. Org. Biomol. Chem. 2017, 15, 4907.

(74) Nishimura, T.; Guo, X.-X.; Hayashi, T. Chem. Asian J. 2008, 3, 1505

(75) Wang, Z.-X.; Bai, X.-Y.; Yao, H.-C.; Li, B.-J. J. Am. Chem. Soc. 2016, 138 14872.

(76) (a) Mitsudo, T.; Nakagawa, Y.; Watanabe, K.; Hori, Y.; Misawa, H.; Watanabe, H.; Watanabe, Y. J. Org. Chem. 1985, 50, 565. (b) Mitsudo, T.; Hori, Y.; Watanabe, Y. Bull. Chem. Soc. Jpn. 1986, 59, 3201.

(77) Ahmed, N.; van Lier, J. E. Tetrahedron Lett. 2007, 48, 5723.

(78) Sawano, T.; Ashouri, A.; Nishimura, T.; Hayashi, T. J. Am. Chem. Soc. 2012, 134, 18936

(79) (a) Trost, B.M.; Taft, B. R.; Masters, J. T.; Lumb, J.-P. J. Am. Chem. Soc 2011, 133, 8502. (b) Trost, B. K.; Masters, J. T.; Taft, B. R.; Lumb, J.P. Chem. Sci. 2011, 133, 8502 .

(80) (a) Nielsen, M.; Jacobsen, C. B.; Paixao, M. W.; Holub, N.; Jørgensen, K. A. J. Am. Chem. Soc. 2009, 131, 10581. (b) Paixao, M. W.; Holub, N.; Vila, C.; Nielsen, M.; Jørgensen, K. A. Angew. Chem. Int. Ed. 2009 $48,7338$.

(81) (a) Capreti, N. M. R.; Jurberg, I. D. Org. Lett. 2015, 17, 2490. (b) Jurberg, I. D. Chem. Eur. J. 2017, 23, 9716. 


\section{Biosketches}

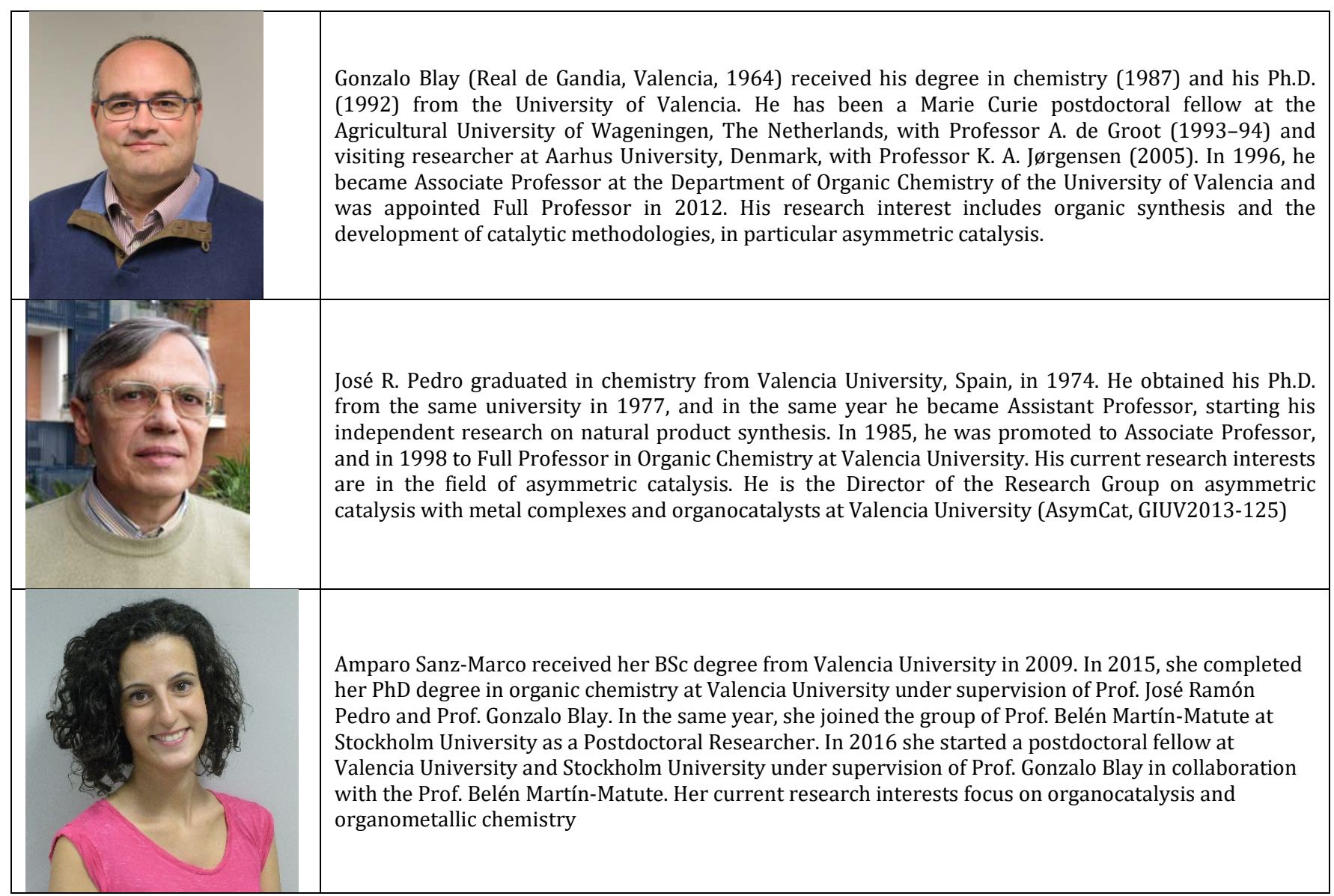

